\title{
Integrability and asymptotic behaviour of a differential-difference matrix equation
}

\author{
Pilar R Gordoa ${ }^{1}$, Andrew Pickering ${ }^{1}$, Jonathan AD Wattis ${ }^{2}$, \\ ${ }^{1}$ Área de Matemática Aplicada, ESCET, Universidad Rey Juan Carlos, \\ C/Tulipán s/n, 28933 Móstoles, Madrid, Spain \\ andrew.pickering@urjc.es pilar.gordoa@urjc.es \\ ${ }^{2}$ School of Mathematical Sciences, University of Nottingham, \\ University Park, Nottingham NG7 2RD, UK \\ Jonathan.Wattis@nottingham.ac.uk
}

\begin{abstract}
In this paper we consider the matrix lattice equation $\mathbf{U}_{n, t}\left(\mathbf{U}_{n+1}-\mathbf{U}_{n-1}\right)=g(n) \mathbf{I}$, in both its autonomous $(g(n)=2)$ and nonautonomous $(g(n)=2 n-1)$ forms. We show that each of these two matrix lattice equations are integrable. In addition, we explore the construction of Miura maps which relate these two lattice equations, via intermediate equations, to matrix analogs of autonomous and nonautonomous Volterra equations, but in two matrix dependent variables. For these last systems, we consider cases where the dependent variables belong to certain special classes of matrices, and obtain integrable coupled systems of autonomous and nonautonomous lattice equations and corresponding Miura maps. Moreover, in the nonautonomous case we present a new integrable nonautonomous matrix Volterra equation, along with its Lax pair. Asymptotic reductions to the matrix potential Korteweg-de Vries and matrix Korteweg-de Vries equations are also given.
\end{abstract}

\section{Highlights}

- We give Miura maps for autonomous and nonautonomous matrix lattice equations and systems.

- We prove the integrability of an autonomous and also of a nonautonomous matrix lattice.

- We obtain integrable multicomponent autonomous and nonautonomous lattices and Miura maps.

- We give a new integrable nonautonomous matrix Volterra equation along with its Lax pair.

- We give asymptotic reductions of matrix lattices to matrix potential $\mathrm{KdV}$ and matrix $\mathrm{KdV}$.

Keywords: Integrability, Asymptotics, matrix equations, differential-difference equations

\section{Introduction}

The aim of the present paper is to undertake a study of the nonautonomous lattice

$$
\mathbf{U}_{n, t}=(2 n-1)\left(\mathbf{U}_{n+1}-\mathbf{U}_{n-1}\right)^{-1},
$$

as well as of its autonomous limit

$$
\mathbf{U}_{n, t}=2\left(\mathbf{U}_{n+1}-\mathbf{U}_{n-1}\right)^{-1}
$$

Equation (1.1) may be derived using the auto-Bäcklund transformations (aBTs) of a certain matrix partial differential equation (PDE), or alternatively those of a matrix second Painlevé $\left(P_{I I}\right)$ equation [1] (see also [2]). The limiting process from (1.1) to (1.2) is presented later.

The scalar case of the autonomous equation (1.2), that is

$$
u_{n, t}=\frac{2}{u_{n+1}-u_{n-1}}
$$


is known to be a completely integrable equation. The transformation

$$
w_{n}=\frac{1}{u_{n+1}-u_{n-1}},
$$

maps solutions of (1.3) to solutions of the equation

$$
w_{n, t}=-2 w_{n}^{2}\left(w_{n+1}-w_{n-1}\right) .
$$

Alternatively, we may regard the system

$$
w_{n}=\frac{1}{u_{n+1}-u_{n-1}}, \quad u_{n, t}=2 w_{n},
$$

as a Bäcklund transformation (BT) between (1.3) and (1.5). Solutions of equation (1.5) may in turn be mapped to solutions of the (rescaled) Volterra lattice

$$
y_{n, t}=-2 y_{n}\left(y_{n+1}-y_{n-1}\right),
$$

using the transformation

$$
y_{n}=w_{n} w_{n+1} .
$$

Equation (1.5) and the Volterra equation (1.7) are well-known completely integrable equations. The above three scalar equations (1.3), (1.5) and (1.7), results on their integrability, relations between them as well as to other known equations, and also various generalisations thereof, can be found in [3] - [21]. In particular, the Miura transformation from (1.5) to (1.7), and the composite Miura transformation from (1.3) to (1.7), can be found for example in [13, 18]. We recall that in [18] the integrability of scalar lattices is shown by constructing Miura transformations to known integrable cases. For example, the existence of a Miura transformation from (a generalisation of) (1.3) to the integrable Volterra equation (1.7) is used to prove the integrability of the former.

One aim of the present paper is to consider to what extent it is possible to extend the above chain of transformations for the scalar autonomous lattice (1.3) to the matrix case (1.2). Here our objective is two-fold: to establish the integrability of (1.2) by extending the mapping (1.4) to the matrix case; and to obtain matrix generalisations of equation (1.7) and the mapping (1.8). A second aim is to consider asymptotic reductions of autonomous matrix lattices to matrix PDEs. These topics are considered in Section 2. In Section 3 we turn our attention to the nonautonomous matrix lattice (1.1), and study the possible extension of the above chain of transformations to this case with the same two-fold objective as in the autonomous case - as well as the asymptotic reduction of nonautonomous matrix lattices to matrix PDEs, and the derivation of autonomous lattices as limiting cases of nonautonomous lattices. We also briefly consider results in the nonautononmous scalar case. In each of sections 2 and 3 a variety of new results are presented. The final section of the paper consists of a discussion and conclusions.

\section{The autonomous matrix lattice}

\subsection{Integrability of the autonomous matrix lattice}

We start by considering the autonomous matrix equation (1.2), that is

$$
\mathbf{U}_{n, t}=2\left(\mathbf{U}_{n+1}-\mathbf{U}_{n-1}\right)^{-1} \text {. }
$$

It can be shown that solutions of this equation are mapped by the transformation

$$
\mathbf{W}_{n}=\left(\mathbf{U}_{n+1}-\mathbf{U}_{n-1}\right)^{-1}
$$

to solutions of the equation

$$
\mathbf{W}_{n, t}=-2 \mathbf{W}_{n}\left(\mathbf{W}_{n+1}-\mathbf{W}_{n-1}\right) \mathbf{W}_{n} .
$$


As with the scalar case (1.6), we may regard the system

$$
\mathbf{W}_{n}=\left(\mathbf{U}_{n+1}-\mathbf{U}_{n-1}\right)^{-1}, \quad \mathbf{U}_{n, t}=2 \mathbf{W}_{n},
$$

as a BT between equations (2.1) and (2.3). Let the shift $(E)$ and difference $(\Delta)$ operators be defined by

$$
E f_{n}=f_{n+1}, \quad \Delta=E-E^{-1},
$$

so $E^{k} f_{n}=f_{n+k}$ for any integer $k$ and $\Delta f_{n}=f_{n+1}-f_{n-1}$. The matrix equation (2.3) has the Lax pair

$$
E \phi_{n}=F_{n} \phi_{n}, \quad \phi_{n, t}=G_{n} \phi_{n},
$$

where

$$
F_{n}=\left(\begin{array}{cc}
2 \lambda \mathbf{W}_{n}^{-1} & \mathbf{I} \\
-\mathbf{I} & \mathbf{0}
\end{array}\right), \quad G_{n}=-4 \lambda\left(\begin{array}{cc}
\lambda \mathbf{I} & \mathbf{W}_{n} \\
-\mathbf{W}_{n-1} & -\lambda \mathbf{I}
\end{array}\right)
$$

and $\mathbf{I}$ is the identity matrix. The compatibility condition of the system (2.6),

$$
0=F_{n, t}+F_{n} G_{n}-G_{n+1} F_{n},
$$

with the definitions (2.7), yields (2.3). Thus equation (2.3) is integrable, and we may deduce - as in the classifications of scalar autonomous lattice equations in [18] - that equation (2.1) is also integrable since it is related to (2.3) by the Miura-type transformation (2.2). We note that (2.3) is a special case of the Jordan-algebraic multi-component generalisations of (1.5) given in [13] along with their Lax pairs, and that the Lax pair (2.6), (2.7) readily gives that for the scalar case (1.5). We note in addition that equation (2.3) can be found in [20] (see also references therein).

The Miura map (2.2) from (2.1) to (2.3) is a matrix version of (1.4) from (1.3) to (1.5). In the next section we turn to the question of matrix generalisations of (1.7) and (1.8): we are unaware of a matrix version of the Miura map (1.8), even though it is straightforward to write down a possible matrix version of (1.7). We note the remark made in [13] that (1.8) is an example of a scalar transformation which is lacking in the multi-component case, and that (1.7) does not have natural multi-component analogs corresponding to Jordan algebraic structures.

\subsection{Matrix Volterra systems}

Let us now consider this question of generalising the transformation (1.8) in order to obtain a mapping from solutions of (2.3) to solutions of a matrix analog of the Volterra equation (1.7). However, this process is complicated by the noncommutativity of matrix multiplication, and the most straightforward matrix generalisation of (1.7) need not in fact be our sought-after matrix analog of (1.7). We define the two products

$$
\mathbf{Y}_{n}=\mathbf{W}_{n} \mathbf{W}_{n+1}, \quad \mathbf{Z}_{n}=\mathbf{W}_{n+1} \mathbf{W}_{n},
$$

which imply

$$
\begin{aligned}
& \mathbf{Y}_{n, t}=-2\left(\mathbf{Y}_{n} \mathbf{Z}_{n+1}-\mathbf{Z}_{n-1} \mathbf{Y}_{n}\right), \\
& \mathbf{Z}_{n, t}=-2\left(\mathbf{Y}_{n+1} \mathbf{Z}_{n}-\mathbf{Z}_{n} \mathbf{Y}_{n-1}\right) .
\end{aligned}
$$

That is, solutions of (2.3) are mapped to solutions of the system (2.10), (2.11). We note, however, that in the system (2.10), (2.11), the entries of the matrices $\mathbf{Y}_{n}$ and $\mathbf{Z}_{n}$ will be subject to constraints. For example, if the entries of a $k \times k$ matrix $\mathbf{W}_{n}$ constitute a set of $k^{2}$ independent functions, so the $\mathbf{W}$-system (2.3) has $k^{2}$ degrees of freedom, then the system (2.10), (2.11) will also have $k^{2}$ - rather than $2 k^{2}$ - degrees of freedom, and so the entries of $\mathbf{Y}_{n}$ and $\mathbf{Z}_{n}$ will be subject to $k^{2}$ constraints. Such restrictions on the dependent variables of the system (2.10), (2.11) are important for an understanding of its relationship to equation (2.3) under the transformation (2.9).

As an example of the constraints that may be required, let us consider the case where

$$
\mathbf{W}_{n}=\left(\begin{array}{cc}
a_{n} & b_{n} \\
0 & c_{n}
\end{array}\right) .
$$


Equation (2.3) is then written

$$
\left(\begin{array}{cc}
a_{n, t} & b_{n, t} \\
0 & c_{n, t}
\end{array}\right)=-2\left(\begin{array}{cc}
a_{n}^{2} \Delta a_{n} & a_{n} b_{n} \Delta a_{n}+a_{n} c_{n} \Delta b_{n}+b_{n} c_{n} \Delta c_{n} \\
0 & c_{n}^{2} \Delta c_{n}
\end{array}\right)
$$

where the operator $\Delta$ is defined as in (2.5). In general matrices of the form (2.12) do not commute, and so the matrices $\mathbf{Y}_{n}=\mathbf{W}_{n} \mathbf{W}_{n+1}$ and $\mathbf{Z}_{n}=\mathbf{W}_{n+1} \mathbf{W}_{n}$ (2.9) are distinct: we have

$$
\mathbf{Y}_{n}=\left(\begin{array}{cc}
u_{n} & y_{n} \\
0 & v_{n}
\end{array}\right), \quad \mathbf{Z}_{n}=\left(\begin{array}{cc}
u_{n} & z_{n} \\
0 & v_{n}
\end{array}\right), \quad \begin{array}{ll}
u_{n}=a_{n} a_{n+1}, & y_{n}=a_{n} b_{n+1}+b_{n} c_{n+1}, \\
v_{n}=c_{n} c_{n+1}, & z_{n}=a_{n+1} b_{n}+b_{n+1} c_{n} .
\end{array}
$$

Whilst the expressions for $y_{n}$ and $z_{n}$ are similar, in general $y_{n} \neq z_{n}$. So the transformation (2.9) maps the system (2.13) in the three variables $\left(a_{n}(t), b_{n}(t), c_{n}(t)\right)$ to the system $(2.10),(2.11)$ in the four variables $\left(u_{n}(t), v_{n}(t), y_{n}(t), z_{n}(t)\right)$, that is,

$$
\begin{array}{ll}
u_{n, t}=-2 u_{n}\left(u_{n+1}-u_{n-1}\right), & y_{n, t}=-2 y_{n}\left(v_{n+1}-u_{n-1}\right)-2 u_{n} z_{n+1}+2 v_{n} z_{n-1}, \\
v_{n, t}=-2 v_{n}\left(v_{n+1}-v_{n-1}\right), & z_{n, t}=-2 z_{n}\left(u_{n+1}-v_{n-1}\right)-2 v_{n} y_{n+1}+2 u_{n} y_{n-1} .
\end{array}
$$

Thus, there must be a consistency condition between the four variables $\left(u_{n}, v_{n}, y_{n}, z_{n}\right)$. From (2.14) note that

$$
a_{n+1} y_{n}=a_{n+1} a_{n} b_{n+1}+a_{n+1} b_{n} c_{n+1}=u_{n} b_{n+1}+c_{n+1}\left(z_{n}-b_{n+1} c_{n}\right)=b_{n+1}\left(u_{n}-v_{n}\right)+c_{n+1} z_{n},
$$

and

$$
c_{n} y_{n}=a_{n} b_{n+1} c_{n}+b_{n} c_{n} c_{n+1}=a_{n}\left(z_{n}-a_{n+1} b_{n}\right)+b_{n} v_{n}=a_{n} z_{n}+b_{n}\left(v_{n}-u_{n}\right) .
$$

We thus obtain

$$
b_{n+1}=\frac{a_{n+1} y_{n}-c_{n+1} z_{n}}{u_{n}-v_{n}}, \quad b_{n}=\frac{a_{n} z_{n}-c_{n} y_{n}}{u_{n}-v_{n}}, \quad b_{n+1}=\frac{a_{n+1} z_{n+1}-c_{n+1} y_{n+1}}{u_{n+1}-v_{n+1}},
$$

where the first two formulae are obtained from (2.17) and (2.18), and the third formula is a shift of the second. Equating the two expressions for $b_{n+1}$ gives

$$
a_{n+1}\left(\frac{y_{n}}{u_{n}-v_{n}}-\frac{z_{n+1}}{u_{n+1}-v_{n+1}}\right)=c_{n+1}\left(\frac{z_{n}}{u_{n}-v_{n}}-\frac{y_{n+1}}{u_{n+1}-v_{n+1}}\right),
$$

hence

$$
\frac{a_{n+1}}{c_{n+1}}=\frac{z_{n}\left(u_{n+1}-v_{n+1}\right)-y_{n+1}\left(u_{n}-v_{n}\right)}{y_{n}\left(u_{n+1}-v_{n+1}\right)-z_{n+1}\left(u_{n}-v_{n}\right)}, \quad \frac{a_{n}}{c_{n}}=\frac{z_{n-1}\left(u_{n}-v_{n}\right)-y_{n}\left(u_{n-1}-v_{n-1}\right)}{y_{n-1}\left(u_{n}-v_{n}\right)-z_{n}\left(u_{n-1}-v_{n-1}\right)},
$$

where, as in (2.19), the second expression is a shift of the first. Taking the product of these two formulae yields

$$
\frac{u_{n}}{v_{n}}=\frac{\left[z_{n}\left(u_{n+1}-v_{n+1}\right)-y_{n+1}\left(u_{n}-v_{n}\right)\right]\left[z_{n-1}\left(u_{n}-v_{n}\right)-y_{n}\left(u_{n-1}-v_{n-1}\right)\right]}{\left[y_{n}\left(u_{n+1}-v_{n+1}\right)-z_{n+1}\left(u_{n}-v_{n}\right)\right]\left[y_{n-1}\left(u_{n}-v_{n}\right)-z_{n}\left(u_{n-1}-v_{n-1}\right)\right]},
$$

which is the required consistency condition on $\left(u_{n}, v_{n}, y_{n}, z_{n}\right)$. This constraint means that the system in $\left(u_{n}, v_{n}, y_{n}, z_{n}\right)$, that is, (2.15) - (2.16), corresponding to (2.13) under the mapping (2.9), is in fact three-dimensional. We note that the expression (2.22) has no explicit dependence on $\left(a_{n}, b_{n}, c_{n}\right)$.

We believe that the mapping (2.9) from equation (2.3) to the system (2.10), (2.11) is new. It is this system (2.10), (2.11) - along with any associated consistency conditions on the entries of the $k \times k$ matrices $\mathbf{Y}_{n}$ and $\mathbf{Z}_{n}$ implied by the mapping (2.9) - that is our sought-after matrix analog of (1.7). In the following two subsections, we consider two special subcases of the mapping (2.9) and the system (2.10), (2.11), defined in terms of properties of the solutions $\mathbf{W}_{n}$ of (2.3). We believe that a general discussion of these two special subcases is also new. 


\subsubsection{Symmetric $\mathbf{W}_{n}$}

If we assume that the matrices $\mathbf{W}_{n}$ are symmetric (that is, $\mathbf{W}_{n}^{T}=\mathbf{W}_{n}$ ), then we find $\mathbf{Z}_{n}^{T}=\mathbf{Y}_{n}$, since $\mathbf{Z}_{n}^{T}=\left(\mathbf{W}_{n+1} \mathbf{W}_{n}\right)^{T}=\mathbf{W}_{n}^{T} \mathbf{W}_{n+1}^{T}=\mathbf{W}_{n} \mathbf{W}_{n+1}=\mathbf{Y}_{n}$. (Note, however, that the symmetry of $\mathbf{W}_{n}$ does not imply that $\mathbf{Y}_{n}=\mathbf{W}_{n} \mathbf{W}_{n+1}$ is symmetric.) Using the relation $\mathbf{Z}_{n}=\mathbf{Y}_{n}^{T}$ to replace $\mathbf{Z}_{n}$ in the system (2.10), (2.11) yields the single equation

$$
\mathbf{Y}_{n, t}=-2\left(\mathbf{Y}_{n} \mathbf{Y}_{n+1}^{T}-\mathbf{Y}_{n-1}^{T} \mathbf{Y}_{n}\right)
$$

since (2.11) becomes the transpose of (2.10). We note once again, however, that there will be constraints on the entries of $\mathbf{Y}_{n}$. For example, if the entries of a $k \times k$ symmetric matrix $\mathbf{W}_{n}$ constitute a set of $k(k+1) / 2$ independent functions, so the $\mathbf{W}$-system (2.3) has $k(k+1) / 2$ degrees of freedom, then the entries of $\mathbf{Y}_{n}$ will be subject to constraints so that equation (2.23) also has $k(k+1) / 2$ degrees of freedom. Such restrictions on the dependent variables of equation (2.23) are important for an understanding of its relationship to equation (2.3) via the transformation $\mathbf{Y}_{n}=\mathbf{W}_{n} \mathbf{W}_{n+1}$.

\subsubsection{Commuting $\mathbf{W}_{n}$}

Let us assume that for all integers $n$ and $m$, the matrices $\mathbf{W}_{n}$ and $\mathbf{W}_{m}$ commute. Then $\mathbf{Z}_{n}=\mathbf{Y}_{n}$ and equations (2.10) and (2.11) give rise to

$$
\mathbf{Y}_{n, t}=-2\left(\mathbf{Y}_{n} \mathbf{Y}_{n+1}-\mathbf{Y}_{n-1} \mathbf{Y}_{n}\right)
$$

and

$$
\mathbf{Y}_{n, t}=-2\left(\mathbf{Y}_{n+1} \mathbf{Y}_{n}-\mathbf{Y}_{n} \mathbf{Y}_{n-1}\right),
$$

respectively. However, since if all matrices $\mathbf{W}_{n}, \mathbf{W}_{m}$ commute then all matrices $\mathbf{Y}_{n}, \mathbf{Y}_{m}$ must also commute, we obtain in fact the single equation

$$
\mathbf{Y}_{n, t}=-2 \mathbf{Y}_{n}\left(\mathbf{Y}_{n+1}-\mathbf{Y}_{n-1}\right)
$$

from both (2.24) and (2.25).

We remark that for any matrix $\mathbf{Y}_{n}$, that is, whether or not all $\mathbf{Y}_{n}$ and $\mathbf{Y}_{m}$ commute, equation (2.25) has the Lax pair (see equations (2.6) and (2.8))

$$
E \phi_{n}=\left(\begin{array}{cc}
\mathbf{I} & \mathbf{Y}_{n} \\
\lambda^{-1} \mathbf{I} & \mathbf{0}
\end{array}\right) \phi_{n}, \quad \phi_{n, t}=2\left(\begin{array}{cc}
-\mathbf{Y}_{n} & \lambda \mathbf{Y}_{n} \\
\mathbf{I} & -\mathbf{Y}_{n-1}-\lambda \mathbf{I}
\end{array}\right) \phi_{n},
$$

and so is integrable. A Lax pair for (2.24), again for any matrix $\mathbf{Y}_{n}$, is given by replacing $\mathbf{Y}_{n}$ with $\mathbf{Y}_{n}^{T}$ in (2.27) (since this will have as compatibility condition equation (2.25) in $\mathbf{Y}_{n}^{T}$, the transpose of which then gives equation (2.24)). The Lax pair (2.27) readily reduces to the well-known Lax pair for the scalar Volterra equation (1.7). Equations (2.24) and (2.25) are known matrix generalisations of the Volterra equation (1.7), and can be found for example in [20] (see also references therein).

From the above we see that we also have a Lax pair for the special case (2.26). This special case is the equation satisfied by the combination $\mathbf{Y}_{n}=\mathbf{W}_{n} \mathbf{W}_{n+1}$ of solutions $\mathbf{W}_{n}$ of (2.3) having the property that for all integers $n$ and $m$ the matrices $\mathbf{W}_{n}$ and $\mathbf{W}_{m}$ commute. We remark that although all such solutions $\mathbf{Y}_{n}$ of (2.26) also have the property that all $\mathbf{Y}_{n}, \mathbf{Y}_{m}$ commute, such a construction still allows us to obtain nontrivial integrable coupled systems of lattice equations. For the corresponding special case of (2.3), with solutions $\mathbf{W}_{n}$ such that any $\mathbf{W}_{n}$ and $\mathbf{W}_{m}$ commute, a Lax pair is provided by equations (2.6) and (2.7). This then yields integrable coupled systems of lattice equations related to those given by (2.26) by transformations obtained from $\mathbf{Y}_{n}=\mathbf{W}_{n} \mathbf{W}_{n+1}$.

As an example let us consider the special case of $2 \times 2$ matrices,

$$
\mathbf{W}_{n}=\left(\begin{array}{ll}
a_{n} & b_{n} \\
c_{n} & d_{n}
\end{array}\right)
$$


The commutativity condition $\mathbf{W}_{n} \mathbf{W}_{n+1}=\mathbf{W}_{n+1} \mathbf{W}_{n}$ gives rise to the equations

$$
b_{n} c_{n+1}=b_{n+1} c_{n}, \quad a_{n} b_{n+1}+b_{n} d_{n+1}=a_{n+1} b_{n}+b_{n+1} d_{n}, \quad c_{n} a_{n+1}+d_{n} c_{n+1}=c_{n+1} a_{n}+d_{n+1} c_{n} .
$$

Up to transposition (note that $\mathbf{W}_{n} \rightarrow \mathbf{W}_{n}^{T}$ is a symmetry of (2.3)), and excluding the trivial case $c_{n}=b_{n}=0$ which gives $\mathbf{W}_{n}$ diagonal and leads to uncoupled systems, equations (2.29) lead us to

$$
\mathbf{W}_{n}=\left(\begin{array}{cc}
a_{n} & b_{n} \\
K b_{n} & a_{n}+M b_{n}
\end{array}\right)
$$

where $K$ and $M$ are arbitrary functions of $t$ (in Appendix A we undertake a further discussion of the derivation of commuting matrices). We may obtain (2.30) by writing the first equation in (2.29) as

$$
(E-1)\left(\frac{c_{n}}{b_{n}}\right)=0,
$$

which gives $c_{n} / b_{n}=K$ and so $c_{n}=K b_{n}$. The third equation in (2.29) then reduces to the second, and this second we solve similarly to the first to obtain $d_{n}-a_{n}=M b_{n}$.

We note that if $\mathbf{W}_{n}$ is of the form (2.30), then all $\mathbf{W}_{n}$ and $\mathbf{W}_{m}$ commute, and so all $\mathbf{Y}_{n}$ and $\mathbf{Y}_{m}$ also commute (where $\mathbf{Y}_{n}$ is given by $\mathbf{Y}_{n}=\mathbf{W}_{n} \mathbf{W}_{n+1}$ ). We also note that $\mathbf{Y}_{n}$ is then given by

$$
\mathbf{Y}_{n}=\left(\begin{array}{cc}
r_{n} & s_{n} \\
K s_{n} & r_{n}+M s_{n}
\end{array}\right)
$$

where

$$
r_{n}=a_{n} a_{n+1}+K b_{n} b_{n+1}, \quad s_{n}=a_{n} b_{n+1}+a_{n+1} b_{n}+M b_{n} b_{n+1} .
$$

Equation (2.3) may then be written as

$$
\mathbf{W}_{n, t}=-2 \mathbf{W}_{n}^{2}\left(\mathbf{W}_{n+1}-\mathbf{W}_{n-1}\right) .
$$

This and equation (2.26), which, using the operator $\Delta$ introduced in (2.5), we rewrite as

$$
\mathbf{W}_{n, t}=-2 \mathbf{W}_{n}^{2} \Delta \mathbf{W}_{n} \quad \text { and } \quad \mathbf{Y}_{n, t}=-2 \mathbf{Y}_{n} \Delta \mathbf{Y}_{n}
$$

where $\mathbf{W}_{n}$ and $\mathbf{Y}_{n}$ are given by (2.30) and (2.32), then give the coupled systems

$$
\begin{aligned}
& a_{n, t}=-2\left(a_{n}^{2}+K b_{n}^{2}\right) \Delta a_{n}-2 K\left(2 a_{n} b_{n}+M b_{n}^{2}\right) \Delta b_{n}, \\
& b_{n, t}=-2\left(a_{n}^{2}+K b_{n}^{2}\right) \Delta b_{n}-2\left(2 a_{n} b_{n}+M b_{n}^{2}\right)\left(\Delta a_{n}+M \Delta b_{n}\right),
\end{aligned}
$$

and

$$
\begin{aligned}
& r_{n, t}=-2 r_{n} \Delta r_{n}-2 K s_{n} \Delta s_{n}, \\
& s_{n, t}=-2 r_{n} \Delta s_{n}-2 s_{n} \Delta r_{n}-2 M s_{n} \Delta s_{n},
\end{aligned}
$$

respectively. In the above coupled systems in $\left(a_{n}(t), b_{n}(t)\right)$ and $\left(r_{n}(t), s_{n}(t)\right), K$ and $M$ are now both constant, as is required by the consistency of each of the entries of the second row in each equation of (2.35). Equation (2.33) maps solutions of the coupled system in $\left(a_{n}(t), b_{n}(t)\right)$ to solutions of the coupled system in $\left(r_{n}(t), s_{n}(t)\right)$. Since (2.6), (2.7) provide us with a Lax pair for the coupled system in $\left(a_{n}(t), b_{n}(t)\right)$, and (2.27) provides us with a Lax pair for the coupled system in $\left(r_{n}(t), s_{n}(t)\right)$, each of these coupled systems is integrable.

We may recover standard examples of commutative $2 \times 2$ matrices as follows:

Elliptic/complex case. We set $K=-1$ and $M=0$ to obtain

$$
\mathbf{W}_{n}=\left(\begin{array}{cc}
a_{n} & b_{n} \\
-b_{n} & a_{n}
\end{array}\right), \quad \mathbf{Y}_{n}=\left(\begin{array}{cc}
r_{n} & s_{n} \\
-s_{n} & r_{n}
\end{array}\right),
$$


with the corresponding systems of equations in $\left(a_{n}, b_{n}\right)$ and $\left(r_{n}, s_{n}\right)$, being given by

$$
\begin{aligned}
a_{n, t} & =2\left(b_{n}^{2}-a_{n}^{2}\right) \Delta a_{n}+4 a_{n} b_{n} \Delta b_{n}, & b_{n, t} & =2\left(b_{n}^{2}-a_{n}^{2}\right) \Delta b_{n}-4 a_{n} b_{n} \Delta a_{n}, \\
r_{n, t} & =-2 r_{n} \Delta r_{n}+2 s_{n} \Delta s_{n}, & s_{n, t} & =-2 r_{n} \Delta s_{n}-2 s_{n} \Delta r_{n} .
\end{aligned}
$$

These are several special cases of (2.36) and (2.37), with the transformation between these pairs of variables being given by the corresponding special case of (2.33), that is, $r_{n}=a_{n} a_{n+1}-b_{n} b_{n+1}$ and $s_{n}=a_{n} b_{n+1}+a_{n+1} b_{n}$.

Dual/shear case. We set $K=0$ and $M=0$ to obtain

$$
\mathbf{W}_{n}=\left(\begin{array}{cc}
a_{n} & b_{n} \\
0 & a_{n}
\end{array}\right), \quad \mathbf{Y}_{n}=\left(\begin{array}{cc}
r_{n} & s_{n} \\
0 & r_{n}
\end{array}\right),
$$

with the corresponding systems of equations in $\left(a_{n}, b_{n}\right)$ and $\left(r_{n}, s_{n}\right)$ being given by

$$
\begin{aligned}
a_{n, t} & =-2 a_{n}^{2} \Delta a_{n}, \quad b_{n, t}=-2 a_{n}^{2} \Delta b_{n}-4 a_{n} b_{n} \Delta a_{n}, \\
r_{n, t} & =-2 r_{n} \Delta r_{n}, \quad s_{n, t}=-2 s_{n} \Delta r_{n}-2 r_{n} \Delta s_{n},
\end{aligned}
$$

and the transformation between these pairs of variables by $r_{n}=a_{n} a_{n+1}$ and $s_{n}=a_{n} b_{n+1}+a_{n+1} b_{n}$. Hyperbolic/cyclic case. We set $K=1, M=0$, to obtain

$$
\mathbf{W}_{n}=\left(\begin{array}{ll}
a_{n} & b_{n} \\
b_{n} & a_{n}
\end{array}\right), \quad \mathbf{Y}_{n}=\left(\begin{array}{ll}
r_{n} & s_{n} \\
s_{n} & r_{n}
\end{array}\right),
$$

with the corresponding systems of equations in $\left(a_{n}, b_{n}\right)$ and $\left(r_{n}, s_{n}\right)$, being given by

$$
\begin{aligned}
a_{n, t} & =-2\left(a_{n}^{2}+b_{n}^{2}\right) \Delta a_{n}-4 a_{n} b_{n} \Delta b_{n}, & b_{n, t} & =-2\left(a_{n}^{2}+b_{n}^{2}\right) \Delta b_{n}-4 a_{n} b_{n} \Delta a_{n}, \\
r_{n, t} & =-2 r_{n} \Delta r_{n}-2 s_{n} \Delta s_{n}, & s_{n, t} & =-2 r_{n} \Delta s_{n}-2 s_{n} \Delta r_{n} .
\end{aligned}
$$

These are special cases of (2.36) and (2.37), with the transformation between these pairs of variables being given by the corresponding special case of (2.33), that is, $r_{n}=a_{n} a_{n+1}+b_{n} b_{n+1}$ and $s_{n}=a_{n} b_{n+1}+a_{n+1} b_{n}$.

A generalised hyperbolic case. We set $K=1, M=-2, a_{n}=e_{n}+f_{n}$ and $b_{n}=f_{n}$ to obtain

$$
\mathbf{W}=\left(\begin{array}{cc}
e_{n}+f_{n} & f_{n} \\
f_{n} & e_{n}-f_{n}
\end{array}\right) .
$$

The corresponding system of equations in $e_{n}$ and $f_{n}$ is

$$
\begin{aligned}
& e_{n, t}=-2\left(e_{n}^{2}+2 f_{n}^{2}\right) \Delta e_{n}-8 e_{n} f_{n} \Delta f_{n}, \\
& f_{n, t}=-4 e_{n} f_{n} \Delta e_{n}-2\left(e_{n}^{2}+2 f_{n}^{2}\right) \Delta f_{n} .
\end{aligned}
$$

With $r_{n}=y_{n}+z_{n}$ and $s_{n}=z_{n}$ we obtain

$$
\mathbf{Y}_{n}=\left(\begin{array}{cc}
y_{n}+z_{n} & z_{n} \\
z_{n} & y_{n}-z_{n}
\end{array}\right)
$$

and corresponding system of equations

$$
y_{n, t}=-2 y_{n} \Delta y_{n}-4 z_{n} \Delta z_{n}, \quad z_{n, t}=-2 z_{n} \Delta y_{n}-2 y_{n} \Delta z_{n} .
$$

The transformation between $\left(e_{n}, f_{n}\right)$ and $\left(y_{n}, z_{n}\right)$, namely

$$
y_{n}=e_{n} e_{n+1}+2 f_{n} f_{n+1}, \quad z_{n}=e_{n} f_{n+1}+e_{n+1} f_{n},
$$

follows from that between $\left(a_{n}, b_{n}\right)$ and $\left(r_{n}, s_{n}\right)$, that is, (2.33).

For a discussion of higher-dimensional commuting matrices, see Appendix A. 


\subsection{Asymptotic reduction of the autonomous U-equation to matrix potential $\mathrm{KdV}$}

We consider the matrix autonomous equation (2.1), which we write as

$$
2 \mathbf{I}=\mathbf{U}_{n, t} \Delta \mathbf{U}_{n}=\mathbf{U}_{n, t}(t)\left[\mathbf{U}_{n+1}(t)-\mathbf{U}_{n-1}(t)\right] .
$$

We use asymptotic techniques to approximate long waves introducing a long spatial scale $(y)$, corresponding to large values of $n$, that is, $n=O\left(h^{-1}\right)$ with $h \ll 1$, and a fast timescale $(\tau)$ via

$$
y=h n, \quad \tau=h^{\sigma} t, \quad \mathbf{U}(x, t)=\widetilde{\mathbf{U}}(y, \tau)=\mathbf{U}_{0}(y, \tau)+h^{2} \mathbf{U}_{1}(y, \tau)+h^{4} \mathbf{U}_{2}(y, \tau) \ldots
$$

Here we have expanded the long-wave solution $(\mathbf{U})$ as a power series in the small parameter $h$. For these asymptotic calculations, we make no assumptions about commutativity or symmetry of $\mathbf{U}_{j}$. The spatial difference of $f(n)=\widehat{f}(y)$ is expanded in terms of derivatives

$$
\Delta f(n)=\widehat{f}(y+h)-\widehat{f}(y-h)=2 h \widehat{f^{\prime}}(y)+\frac{2}{6} h^{3} \widehat{f}^{\prime \prime \prime}(y)+\ldots
$$

Expanding all quantites in (2.48), we find

$$
\begin{gathered}
2 \mathbf{I}=2 h^{\sigma+1}\left(\mathbf{U}_{0, \tau}+h^{2} \mathbf{U}_{1, \tau}+h^{4} \mathbf{U}_{2, \tau}+\ldots\right)\left(\mathbf{U}_{0, y}+h^{2} \mathbf{U}_{1, y}+h^{4} \mathbf{U}_{2, y}\right. \\
\left.+\frac{1}{6} h^{2} \mathbf{U}_{0, y y y}+\frac{1}{6} h^{4} \mathbf{U}_{1, y y y}+\frac{1}{120} h^{4} \mathbf{U}_{0, y y y y y}+\ldots\right) .
\end{gathered}
$$

Before equating terms of equal order in $h$, we transform to a moving coordinate frame and introduce a new longer timescale via

$$
z=y-c \tau, \quad T=h^{2} \tau, \quad \partial_{y}=\partial_{z}, \quad \partial_{\tau}=\partial_{\tau}-c \partial_{z}+h^{2} \partial_{T},
$$

so that $(2.51)$ becomes

$$
\begin{aligned}
\mathbf{I}= & h^{\sigma+1}\left(\mathbf{U}_{0, \tau}-c \mathbf{U}_{0, z}+h^{2} \mathbf{U}_{0, T}+h^{2} \mathbf{U}_{1, \tau}-c h^{2} \mathbf{U}_{1, z}+h^{4} \mathbf{U}_{1, T}+h^{4} \mathbf{U}_{2, \tau}-c h^{4} \mathbf{U}_{2, z}+\ldots\right) \\
& \times\left(\mathbf{U}_{0, z}+h^{2} \mathbf{U}_{1, z}+h^{4} \mathbf{U}_{2, z}+\frac{1}{6} h^{2} \mathbf{U}_{0, z z z}+\frac{1}{6} h^{4} \mathbf{U}_{1, z z z}+\frac{1}{120} h^{4} \mathbf{U}_{0, z z z z z}+\ldots\right) .
\end{aligned}
$$

Expanding and equating leading order terms, we find

$$
\mathbf{I}=h^{1+\sigma}\left(\mathbf{U}_{0, \tau}-c \mathbf{U}_{0, z}\right) \mathbf{U}_{0, z},
$$

hence we assign $\sigma=-1$ and choose

$$
\mathbf{U}_{0}=z \mathbf{K}+\tau \mathbf{M}+\mathbf{L}(T), \quad \mathbf{M}=c \mathbf{K}+\mathbf{K}^{-1},
$$

where $\mathbf{K}$ is an arbitrary constant matrix, and $\mathbf{L}(T)$ is an arbitrary matrix function of $T$. For simplicity, and to obtain the most general form of solution for the higher order term $\mathbf{U}_{1}$, we take $\mathbf{K}=k \mathbf{I}$, so that $\mathbf{M}=\left(c k+k^{-1}\right) \mathbf{I}$.

\subsubsection{First correction terms}

The first correction terms from (2.53) imply

$$
\mathbf{0}=\left(\mathbf{U}_{0, \tau}-c \mathbf{U}_{0, z}\right)\left(\mathbf{U}_{1, z}+\frac{1}{6} \mathbf{U}_{0, z z z}\right)+\left(\mathbf{U}_{0, T}+\mathbf{U}_{1, \tau}-c \mathbf{U}_{1, z}\right) \mathbf{U}_{0, z},
$$

from which, using the leading order solution (2.55), we find

$$
\mathbf{0}=k^{-2} \mathbf{U}_{1, z}+\mathbf{L}^{\prime}(T)+\mathbf{U}_{1, \tau}-c \mathbf{U}_{1, z} .
$$

From this equation, we determine the speed of the wave, $c$, leaving $\mathbf{U}_{1}$ to be determined from higher order equations. We assume $\mathbf{L}^{\prime}(T)=0$ so there is no evolution of $\mathbf{U}_{0}$ on the long timescale; also $\mathbf{U}_{1, \tau}=\mathbf{0}$, so that $\mathbf{U}_{1}$ has no $\tau$-dependence. Then, from the coefficients of $\mathbf{U}_{1, z}$, we find $c=k^{-2}$. 


\subsubsection{Second correction terms}

The second correction terms in (2.53) yield

$$
\begin{aligned}
\mathbf{0}= & \left(\mathbf{U}_{0, \tau}-c \mathbf{U}_{0, z}\right)\left(\mathbf{U}_{2, z}+\frac{1}{6} \mathbf{U}_{1, z z z}+\frac{1}{120} \mathbf{U}_{0, z z z z z}\right)+\left(\mathbf{U}_{2, \tau}-c \mathbf{U}_{2, z}+\mathbf{U}_{1, T}\right) \mathbf{U}_{0, z} \\
& +\left(\mathbf{U}_{1, \tau}-c \mathbf{U}_{1, z}+\mathbf{U}_{0, T}\right)\left(\mathbf{U}_{1, z}+\frac{1}{6} \mathbf{U}_{0, z z z}\right) ;
\end{aligned}
$$

using the formulae (2.55) for $\mathbf{U}_{0}$, this simplifies to

$$
\mathbf{0}=\frac{1}{6} \mathbf{U}_{1, z z z}-k^{-1} \mathbf{U}_{1, z}^{2}+k^{2} \mathbf{U}_{1, T},
$$

where we have assumed no dependence of $\mathbf{U}_{2}$ on $\tau$. This last equation is the matrix form of the potential $\mathrm{KdV}$ equation; $\mathbf{U}_{1, z}$ satisfies the matrix $\mathrm{KdV}$ equation. Here there are no other restrictions on $\mathbf{U}_{1}$ : it may be asymmetric and we have not imposed any commutativity properties.

\subsection{Asymptotic reduction of the autonomous $\mathbf{W}$-equation to matrix $\mathrm{KdV}$}

To consider the small amplitude behaviour of solutions of (2.3), we introduce a small parameter, $h \ll 1$, writing

$$
\mathbf{W}_{n}(t)=\widetilde{\mathbf{W}}_{0}(z, T)+h^{2} \widetilde{\mathbf{W}}_{1}(z, T)+h^{4} \widetilde{\mathbf{W}}_{2}(z, T)+\ldots
$$

Using the same notation as in (2.50) and (2.52), we obtain

$$
\begin{aligned}
\left(c \widetilde{\mathbf{W}}_{0, z}-\right. & \left.h^{2} \widetilde{\mathbf{W}}_{0, T}+c h^{2} \widetilde{\mathbf{W}}_{1, z}-h^{4} \widetilde{\mathbf{W}}_{1, T}+c h^{4} \widetilde{\mathbf{W}}_{2, z}+\ldots\right) \\
= & 4\left(\widetilde{\mathbf{W}}_{0}+h^{2} \widetilde{\mathbf{W}}_{1}+h^{4} \widetilde{\mathbf{W}}_{2}+\ldots\right)\left(\widetilde{\mathbf{W}}_{0, z}+\frac{1}{6} h^{2} \widetilde{\mathbf{W}}_{0, z z z}+\frac{1}{120} h^{4} \widetilde{\mathbf{W}}_{0, z z z z z}\right. \\
& \left.+h^{2} \widetilde{\mathbf{W}}_{1, z}+\frac{1}{6} h^{4} \widetilde{\mathbf{W}}_{1, z z z}+h^{4} \widetilde{\mathbf{W}}_{2, z}+\ldots\right)\left(\widetilde{\mathbf{W}}_{0}+h^{2} \widetilde{\mathbf{W}}_{1}+h^{4} \widetilde{\mathbf{W}}_{2}+\ldots\right) .
\end{aligned}
$$

At leading order, we find $c \widetilde{\mathbf{W}}_{0, z}=4 \widetilde{\mathbf{W}}_{0} \widetilde{\mathbf{W}}_{0, z} \widetilde{\mathbf{W}}_{0}$, which has the solution $\widetilde{\mathbf{W}}_{0}=\mathbf{K}(T)$.

Using $\widetilde{\mathbf{W}}_{0}=\mathbf{K}(T)$, the first correction terms give

$$
\left(c \widetilde{\mathbf{W}}_{1, z}-\widetilde{\mathbf{W}}_{0, T}\right)=4 \widetilde{\mathbf{W}}_{0} \widetilde{\mathbf{W}}_{1, z} \widetilde{\mathbf{W}}_{0}
$$

which simplifies to

$$
c \widetilde{\mathbf{W}}_{1, z}-\mathbf{K}^{\prime}(T)=4 \mathbf{K}(T) \widetilde{\mathbf{W}}_{1, z} \mathbf{K}(T) .
$$

In the case $\mathbf{K}(T)=k \mathbf{I}$ we have $c=4 k^{2}$, and this equation is trivially satisfied. Since $\widetilde{\mathbf{W}}_{0}$ is then diagonal, it commutes with any other matrix.

The second correction terms give

$$
\left(c \widetilde{\mathbf{W}}_{2, z}-\widetilde{\mathbf{W}}_{1, T}\right)=4 \widetilde{\mathbf{W}}_{0}\left(\widetilde{\mathbf{W}}_{2, z}+\frac{1}{6} \widetilde{\mathbf{W}}_{1, z z z}\right) \widetilde{\mathbf{W}}_{0}+4 \widetilde{\mathbf{W}}_{0} \widetilde{\mathbf{W}}_{1, z} \widetilde{\mathbf{W}}_{1}+4 \widetilde{\mathbf{W}}_{1} \widetilde{\mathbf{W}}_{1, z} \widetilde{\mathbf{W}}_{0}
$$

which, with $\widetilde{\mathbf{W}}_{0}=\mathbf{K}(T)=k \mathbf{I}$ and $\mathbf{W}_{2}=\mathbf{0}$, simplifies to the matrix $\mathrm{KdV}$ equation

$$
\mathbf{0}=\widetilde{\mathbf{W}}_{1, T}+\frac{2}{3} k^{2} \widetilde{\mathbf{W}}_{1, z z z}+4 k \widetilde{\mathbf{W}}_{1, z} \widetilde{\mathbf{W}}_{1}+4 k \widetilde{\mathbf{W}}_{1} \widetilde{\mathbf{W}}_{1, z}
$$

a well-known integrable equation. Here there is no assumption that $\widetilde{\mathbf{W}}_{1}$ is symmetric or has any special properties to allow commutativity with other matrices.

\section{The nonautonomous matrix lattice}

In this section we give results for the nonautonomous matrix lattice (1.1). These results are analogous to those given in the previous section for the autonomous case. In addition, we show how the autonomous case may be derived from the nonautonomous case via a limiting process, and also briefly discuss results for the scalar case of the nonautonomous lattice. 


\subsection{Integrability of the nonautonomous matrix lattice}

Let us consider the nonautonomous matrix equation (1.1), that is

$$
\mathbf{U}_{n, t}=(2 n-1)\left(\mathbf{U}_{n+1}-\mathbf{U}_{n-1}\right)^{-1} \text {. }
$$

Solutions of this equation are mapped by the transformation

$$
\mathbf{W}_{n}=\left(\mathbf{U}_{n+1}-\mathbf{U}_{n-1}\right)^{-1},
$$

to solutions of the equation

$$
\mathbf{W}_{n, t}=-\mathbf{W}_{n}\left[(2 n+1) \mathbf{W}_{n+1}-(2 n-3) \mathbf{W}_{n-1}\right] \mathbf{W}_{n} .
$$

We may alternatively regard the system

$$
\mathbf{W}_{n}=\left(\mathbf{U}_{n+1}-\mathbf{U}_{n-1}\right)^{-1}, \quad \mathbf{U}_{n, t}=(2 n-1) \mathbf{W}_{n},
$$

as a BT between equations (3.1) and (3.3). Equation (3.3) has the nonisospectral Lax pair (see equations (2.6) and (2.8)),

$$
E \phi_{n}=\left(\begin{array}{cc}
2 \lambda(t) \mathbf{W}_{n}^{-1} & \mathbf{I} \\
-\mathbf{I} & \mathbf{0}
\end{array}\right) \phi_{n}, \quad \phi_{n, t}=2 \lambda(t)\left(\begin{array}{cc}
-2(n-1) \lambda(t) \mathbf{I} & (1-2 n) \mathbf{W}_{n} \\
(2 n-3) \mathbf{W}_{n-1} & 2(n-1) \lambda(t) \mathbf{I}
\end{array}\right) \phi_{n},
$$

where $\lambda(t)$ satisfies $\lambda_{t}(t)=-4 \lambda(t)^{3}$, and so is integrable. Thus equation (3.1) is also integrable, as it is related to (3.3) via the Miura-type transformation (3.2).

\subsection{The scalar case}

In the scalar case we obtain the nonautonomous equation

$$
u_{n, t}=\frac{2 n-1}{u_{n+1}-u_{n-1}}
$$

related by the transformation

$$
w_{n}=\frac{1}{u_{n+1}-u_{n-1}},
$$

to the nonautonomous equation

$$
w_{n, t}=-w_{n}^{2}\left[(2 n+1) w_{n+1}-(2 n-3) w_{n-1}\right] .
$$

In addition, the transformation

$$
y_{n}=w_{n} w_{n+1},
$$

maps solutions of (3.8) to solutions of the nonautonomous Volterra equation

$$
y_{n, t}=y_{n}\left[(2 n-3) y_{n-1}-(2 n+3) y_{n+1}-2 y_{n}\right] \text {. }
$$

This equation and its nonisospectral Lax pair can be found in [17] (equation (67) with $\alpha_{1}=1, \beta_{0}=$ 2 and $\beta_{1}=0$ ); nonisospectral Volterra flows have also been discussed for example in $[8,14,16]$. We note that the nonautonomous matrix lattice (3.3) is a special case of Jordan-algebraic multicomponent generalisations of (3.8) given in [13] along with their Lax pairs, and that the Lax pair (3.5) readily gives that for the scalar case (3.8). Moreover, the limiting process to autonomous equations, and the asymptotic reductions of (3.1) and (3.3) to matrix PDEs, as discussed in later subsections, also readily give corresponding results for the case of scalar dependent variables.

We believe the matrix and scalar nonautonomous equations (3.1) and (3.6) derived in [1] (see also [2]), and their relation to (3.3) and (3.8) under the mappings (3.2) and (3.7) respectively, to be new. We also believe the relation between the scalar nonautonomous equations (3.8) and (3.10) via the transformation (3.9) to be new. This mapping relies on the commutativity of scalar functions; we now turn to the question of generalising the transformation (3.9) and equation (3.10) to the matrix case, where (in general) multiplication is noncommutative. At this point we recall once again the remark made in [13], albeit in the autonomous case, that a multi-component version of the transformation (3.9) is lacking, and that the scalar equation in $y_{n}$ does not have natural multi-component analogs corresponding to Jordan algebraic structures. 


\subsection{Nonautonomous matrix Volterra systems}

In order to address the question spelt out above, we proceed as in the autonomous case. We use the definitions (2.9), namely $\mathbf{Y}_{n}=\mathbf{W}_{n} \mathbf{W}_{n+1}, \mathbf{Z}_{n}=\mathbf{W}_{n+1} \mathbf{W}_{n}$, which imply

$$
\begin{aligned}
& \mathbf{Y}_{n, t}=(2 n-3) \mathbf{Z}_{n-1} \mathbf{Y}_{n}-(2 n+3) \mathbf{Y}_{n} \mathbf{Z}_{n+1}-2 \mathbf{Y}_{n}^{2}, \\
& \mathbf{Z}_{n, t}=(2 n-3) \mathbf{Z}_{n} \mathbf{Y}_{n-1}-(2 n+3) \mathbf{Y}_{n+1} \mathbf{Z}_{n}-2 \mathbf{Z}_{n}^{2} .
\end{aligned}
$$

That is, solutions of (3.3) are mapped to solutions of (3.11), (3.12). As in the autonomous case, the mapping (2.9) implies constraints on the entries of the matrices $\mathbf{Y}_{n}$ and $\mathbf{Z}_{n}$. Such restrictions on the dependent variables of the system (3.11), (3.12) are important for an understanding of its relationship to equation (3.3) under the mapping (2.9). It is this system, along with any such consistency conditions, that is our sought-after matrix analog of (3.10). We believe these results to be new. We now consider, as in the autonomous case, two special cases defined in terms of properties of the solutions $\mathbf{W}_{n}$ of (3.3).

\subsubsection{Symmetric $\mathbf{W}_{n}$}

If $\mathbf{W}_{n}$ is symmetric, then from (2.9) we have $\mathbf{Z}_{n}^{T}=\mathbf{W}_{n}^{T} \mathbf{W}_{n+1}^{T}=\mathbf{W}_{n} \mathbf{W}_{n+1}=\mathbf{Y}_{n}$, hence the system (3.11), (3.12) yields the single equation

$$
\mathbf{Y}_{n, t}=(2 n-3) \mathbf{Y}_{n-1}^{T} \mathbf{Y}_{n}-(2 n+3) \mathbf{Y}_{n} \mathbf{Y}_{n+1}^{T}-2 \mathbf{Y}_{n}^{2}
$$

since (3.12) becomes the transpose of (3.11). We note once again, as in the autonomous case, that there will be constraints on the entries of $\mathbf{Y}_{n}$, such restrictions being important for an understanding of the relationship of (3.13) to (3.3) via the transformation $\mathbf{Y}_{n}=\mathbf{W}_{n} \mathbf{W}_{n+1}$.

\subsubsection{Commuting $\mathbf{W}_{n}$}

In the case of commuting matrices $\mathbf{W}_{n}$, that is, such that for all integers $n$ and $m$ the matrices $\mathbf{W}_{n}$ and $\mathbf{W}_{m}$ commute, then $\mathbf{Z}_{n}=\mathbf{Y}_{n}$ and equations (3.11) and (3.12) yield

$$
\begin{aligned}
& \mathbf{Y}_{n, t}=(2 n-3) \mathbf{Y}_{n-1} \mathbf{Y}_{n}-(2 n+3) \mathbf{Y}_{n} \mathbf{Y}_{n+1}-2 \mathbf{Y}_{n}^{2}, \\
& \mathbf{Y}_{n, t}=(2 n-3) \mathbf{Y}_{n} \mathbf{Y}_{n-1}-(2 n+3) \mathbf{Y}_{n+1} \mathbf{Y}_{n}-2 \mathbf{Y}_{n}^{2},
\end{aligned}
$$

respectively. Since if all matrices $\mathbf{W}_{n}, \mathbf{W}_{m}$ commute then so do all matrices $\mathbf{Y}_{n}, \mathbf{Y}_{m}$, we obtain the single equation

$$
\mathbf{Y}_{n, t}=\mathbf{Y}_{n}\left[(2 n-3) \mathbf{Y}_{n-1}-(2 n+3) \mathbf{Y}_{n+1}-2 \mathbf{Y}_{n}\right]
$$

from (3.14) and (3.15).

Let us now consider the nonisospectral Lax pair (see equations (2.6) and (2.8))

$$
E \phi_{n}=\left(\begin{array}{cc}
\mathbf{I} & \mathbf{Y}_{n} \\
\lambda(t)^{-1} \mathbf{I} & \mathbf{0}
\end{array}\right) \phi_{n}, \quad \phi_{n, t}=\left(\begin{array}{cc}
-(2 n-1) \mathbf{Y}_{n}-2 \mathbf{X}_{n} & (2 n+1) \lambda(t) \mathbf{Y}_{n} \\
(2 n-1) \mathbf{I} & -(2 n-3) \mathbf{Y}_{n-1}-2 \mathbf{X}_{n-1}-(2 n+1) \lambda(t) \mathbf{I}
\end{array}\right) \phi_{n},
$$

where $\lambda(t)$ satisfies $\lambda_{t}(t)=2 \lambda_{t}(t)^{2}$, but where no assumptions are made about the commutativity of any matrices $\mathbf{Y}_{n}, \mathbf{Y}_{m}, \mathbf{X}_{n}, \mathbf{X}_{m}$. The compatibility condition of this Lax pair yields the system

$$
\begin{aligned}
\mathbf{Y}_{n, t} & =(2 n-3) \mathbf{Y}_{n} \mathbf{Y}_{n-1}-(2 n+1) \mathbf{Y}_{n+1} \mathbf{Y}_{n}+2\left(\mathbf{Y}_{n} \mathbf{X}_{n-1}-\mathbf{X}_{n+1} \mathbf{Y}_{n}\right), \\
\mathbf{X}_{n+1}-\mathbf{X}_{n} & =\mathbf{Y}_{n+1},
\end{aligned}
$$

which is therefore integrable. This system can be written alternatively as

$$
\begin{aligned}
\mathbf{Y}_{n, t} & =(2 n-3) \mathbf{Y}_{n} \mathbf{Y}_{n-1}-(2 n+3) \mathbf{Y}_{n+1} \mathbf{Y}_{n}-2 \mathbf{Y}_{n}^{2}+2\left[\mathbf{Y}_{n}, \mathbf{X}_{n}\right], \\
\mathbf{X}_{n+1}-\mathbf{X}_{n} & =\mathbf{Y}_{n+1},
\end{aligned}
$$

where the last term on the right-hand-side of (3.20) is (twice) the commutator of $\mathbf{Y}_{n}$ and $\mathbf{X}_{n}$. We believe the matrix system (3.20), (3.21) and its nonisospectral Lax pair (3.17) to be new. 
In the special case where, for all integers $n$ and $m$, all matrices $\mathbf{Y}_{n}, \mathbf{Y}_{m}, \mathbf{X}_{n}$ and $\mathbf{X}_{m}$ commute, the above result gives a Lax pair for equation (3.16) (along with equation (3.21) to be satisfied by the potential $\mathbf{X}_{n}$ ). This special case (3.16) is the equation satisfied by the combination $\mathbf{Y}_{n}=\mathbf{W}_{n} \mathbf{W}_{n+1}$ of solutions $\mathbf{W}_{n}$ of (3.3) having the property that all matrices $\mathbf{W}_{n}$ and $\mathbf{W}_{m}$ commute. We remark that even though all such solutions $\mathbf{Y}_{n}$ of (3.16) have the property that all $\mathbf{Y}_{n}$ and $\mathbf{Y}_{m}$ commute, we are still able to obtain nontrivial integrable coupled systems of nonautonomous lattice equations. For the corresponding special case of (3.3), with solutions $\mathbf{W}_{n}$ such that all $\mathbf{W}_{n}$ and $\mathbf{W}_{m}$ commute, which we may write as

$$
\mathbf{W}_{n, t}=-\mathbf{W}_{n}^{2}\left[(2 n+1) \mathbf{W}_{n+1}-(2 n-3) \mathbf{W}_{n-1}\right],
$$

a Lax pair is provided by (3.5). This then yields integrable coupled systems of nonautonomous lattice equations related to those given by (3.16) by transformations obtained from $\mathbf{Y}_{n}=\mathbf{W}_{n} \mathbf{W}_{n+1}$.

In the autonomous case, whether or not all $\mathbf{Y}_{n}$ and $\mathbf{Y}_{m}$ commute, we were able to give a Lax pair for (2.25) (and for (2.24), equivalent to (2.25) under $\mathbf{Y}_{n} \rightarrow \mathbf{Y}_{n}^{T}$ ). However, in the nonautonomous case, without making the additional assumption that all $\mathbf{Y}_{n}$ and $\mathbf{Y}_{m}$ commute, we are unable to give Lax pairs for equations (3.14) and (3.15). Whereas in the autonomous case, the noncommutative generalisation of (2.26) is the similar-looking (2.25) (or (2.24)), in the nonautonomous case the noncommutative generalisation of (3.16) is the system (3.20), (3.21), or the equivalent system

$$
\begin{aligned}
\mathbf{Y}_{n, t} & =(2 n-3) \mathbf{Y}_{n-1} \mathbf{Y}_{n}-(2 n+3) \mathbf{Y}_{n} \mathbf{Y}_{n+1}-2 \mathbf{Y}_{n}^{2}-2\left[\mathbf{Y}_{n}, \mathbf{X}_{n}\right], \\
\mathbf{X}_{n+1}-\mathbf{X}_{n} & =\mathbf{Y}_{n+1},
\end{aligned}
$$

(note the sign of the last term of (3.23)). A Lax pair for (3.23), (3.24) is given by replacing $\mathbf{Y}_{n}$ and $\mathbf{X}_{n}$ in (3.17) by $\mathbf{Y}_{n}^{T}$ and $\mathbf{X}_{n}^{T}$, since this will then have the system (3.20), (3.21) in $\mathbf{Y}_{n}^{T}$ and $\mathbf{X}_{n}^{T}$ as compatibility condition, the transpose of which then gives the system (3.23), (3.24). We remark in passing that the solution $X_{n}$ of (3.21), or (3.24), may be expressed nonlocally as

$$
\mathbf{X}_{n}=\Gamma(t)+\sum_{j=-\infty}^{n} \mathbf{Y}_{j}
$$

where $\Gamma(t)$ is an arbitrary matrix function of $t$.

As an example, we consider once again the special case of commuting $2 \times 2$ matrices. As in the autonomous case, we obtain the expressions (2.30) and (2.32) for $\mathbf{W}_{n}$ and $\mathbf{Y}_{n}$. Equations (3.22) and (3.16) then give rise to the systems

$$
\begin{aligned}
a_{n, t}= & -\left(a_{n}^{2}+K b_{n}^{2}\right)\left[(2 n+1) a_{n+1}-(2 n-3) a_{n-1}\right]-K b_{n}\left(2 a_{n}+M b_{n}\right)\left[(2 n+1) b_{n+1}-(2 n-3) b_{n-1}\right], \\
b_{n, t}= & -\left(a_{n}^{2}+K b_{n}^{2}\right)\left[(2 n+1) b_{n+1}-(2 n-3) b_{n-1}\right]-b_{n}\left(2 a_{n}+M b_{n}\right)\left[(2 n+1) a_{n+1}-(2 n-3) a_{n-1}\right] \\
& -M b_{n}\left(2 a_{n}+M b_{n}\right)\left[(2 n+1) b_{n+1}-(2 n-3) b_{n-1}\right],
\end{aligned}
$$

and

$$
\begin{aligned}
r_{n, t}= & r_{n}\left[(2 n-3) r_{n-1}-(2 n+3) r_{n+1}-2 r_{n}\right]+K s_{n}\left[(2 n-3) s_{n-1}-(2 n+3) s_{n+1}-2 s_{n}\right], \\
s_{n, t}= & r_{n}\left[(2 n-3) s_{n-1}-(2 n+3) s_{n+1}-2 s_{n}\right]+s_{n}\left[(2 n-3) r_{n-1}-(2 n+3) r_{n+1}-2 r_{n}\right] \\
& +M s_{n}\left[(2 n-3) s_{n-1}-(2 n+3) s_{n+1}-2 s_{n}\right],
\end{aligned}
$$

respectively where $K$ and $M$ are arbitrary constants. Here the pairs of variables $\left(a_{n}, b_{n}\right)$ and $\left(r_{n}, s_{n}\right)$ are related by (2.33) (obtained from $\mathbf{Y}_{n}=\mathbf{W}_{n} \mathbf{W}_{n+1}$ ). Each of the above two systems is integrable: (3.5) gives us a Lax pair for the system in $\left(a_{n}, b_{n}\right)$, and (3.17) gives us a Lax pair for the system in $\left(r_{n}, s_{n}\right)$. We note that in this commutative case, we may take $\Gamma(t)$ in (3.25) to be given by

$$
\Gamma(t)=\left(\begin{array}{cc}
r(t) & s(t) \\
K s(t) & r(t)+M s(t)
\end{array}\right) .
$$

The systems of nonautonomous lattice equations in $\left(a_{n}, b_{n}\right)$ and $\left(r_{n}, s_{n}\right)$, or $\left(e_{n}, f_{n}\right)$ and $\left(y_{n}, z_{n}\right)$, corresponding to standard examples of commutative $2 \times 2$ matrices (elliptic/complex, dual/shear, hyperbolic/cyclic, generalised hyperbolic), as considered previously in the autonomous case, can be found in Appendix B. 


\subsection{Autonomous limits}

Let us consider how to obtain (1.2), (2.3) and the system (2.10), (2.11) as autonomous limits of (3.1), (3.3) and the system (3.11), (3.12), respectively.

If we write

$$
\mathbf{U}_{n}=\mathbf{U}(n, t)=\sqrt{p} \widehat{\mathbf{U}}(m, t), \quad n=m+p,
$$

for any given $p \in \mathbb{N}$, then we obtain from (3.1) a lattice equation in $\widehat{\mathbf{U}}_{m}=\widehat{\mathbf{U}}(m, t)$, given by

$$
\widehat{\mathbf{U}}_{m, t}=\left(\frac{2 m-1}{p}+2\right)\left(\widehat{\mathbf{U}}_{m+1}-\widehat{\mathbf{U}}_{m-1}\right)^{-1} .
$$

In far-field limit when $p \rightarrow \infty$ this then yields

$$
\widehat{\mathbf{U}}_{m, t}=2\left(\widehat{\mathbf{U}}_{m+1}-\widehat{\mathbf{U}}_{m-1}\right)^{-1}
$$

that is, the autonomous lattice (1.2).

Similarly, defining $\widehat{\mathbf{W}}_{m}=\widehat{\mathbf{W}}(m, t)$ via

$$
\mathbf{W}_{n}=\mathbf{W}(n, t)=\frac{1}{\sqrt{p}} \widehat{\mathbf{W}}(m, t), \quad n=m+p,
$$

for any given $p \in \mathbb{N}$, we obtain from (3.3) the equation

$$
\widehat{\mathbf{W}}_{m, t}=-\widehat{\mathbf{W}}_{m}\left[\left(\frac{2 m+1}{p}+2\right) \widehat{\mathbf{W}}_{m+1}-\left(\frac{2 m-3}{p}+2\right) \widehat{\mathbf{W}}_{m-1}\right] \widehat{\mathbf{W}}_{m},
$$

which, in the far-field limit $p \rightarrow \infty$ then gives

$$
\widehat{\mathbf{W}}_{m, t}=-2 \widehat{\mathbf{W}}_{m}\left(\widehat{\mathbf{W}}_{m+1}-\widehat{\mathbf{W}}_{m-1}\right) \widehat{\mathbf{W}}_{m},
$$

that is, the autonomous lattice (2.3).

Finally, defining $\widehat{\mathbf{Y}}_{m}=\widehat{\mathbf{Y}}(m, t)$ and $\widehat{\mathbf{Z}}_{m}=\widehat{\mathbf{Z}}(m, t)$ by

$$
\mathbf{Y}_{n}=\mathbf{Y}(n, t)=\frac{1}{p} \widehat{\mathbf{Y}}(m, t), \quad \mathbf{Z}_{n}=\mathbf{Z}(n, t)=\frac{1}{p} \widehat{\mathbf{Z}}(m, t), \quad n=m+p,
$$

for any given $p \in \mathbb{N}$, we obtain from (3.11) and (3.12) the system

$$
\begin{aligned}
& \widehat{\mathbf{Y}}_{m, t}=\left(\frac{2 m-3}{p}+2\right) \widehat{\mathbf{Z}}_{m-1} \widehat{\mathbf{Y}}_{m}-\left(\frac{2 m+3}{p}+2\right) \widehat{\mathbf{Y}}_{m} \widehat{\mathbf{Z}}_{m+1}-\frac{2}{p} \widehat{\mathbf{Y}}_{m}^{2}, \\
& \widehat{\mathbf{Z}}_{m, t}=\left(\frac{2 m-3}{p}+2\right) \widehat{\mathbf{Z}}_{m} \widehat{\mathbf{Y}}_{m-1}-\left(\frac{2 m+3}{p}+2\right) \widehat{\mathbf{Y}}_{m+1} \widehat{\mathbf{Z}}_{m}-\frac{2}{p} \widehat{\mathbf{Z}}_{m}^{2}
\end{aligned}
$$

When $p \rightarrow \infty$ this system becomes

$$
\begin{aligned}
\widehat{\mathbf{Y}}_{m, t} & =2 \widehat{\mathbf{Z}}_{m-1} \widehat{\mathbf{Y}}_{m}-2 \widehat{\mathbf{Y}}_{m} \widehat{\mathbf{Z}}_{m+1}, \\
\widehat{\mathbf{Z}}_{m, t} & =2 \widehat{\mathbf{Z}}_{m} \widehat{\mathbf{Y}}_{m-1}-2 \widehat{\mathbf{Y}}_{m+1} \widehat{\mathbf{Z}}_{m},
\end{aligned}
$$

that is, the autonomous system (2.10), (2.11).

\subsection{Asymptotic reduction of the nonautonomous $\mathrm{U}$-equation to matrix potential $\mathrm{KdV}$}

We return to the non-autonomous differential-difference equation

$$
(2 n-1) \mathbf{I}=\mathbf{U}_{n, t} \Delta \mathbf{U}_{n},
$$


which was previously studied in [2]. We start by transforming the independent variable $n$ via $2 n-1=2 x$ so that the equation has a more symmetric form $2 x \mathbf{I}=\mathbf{U}_{t} \Delta U$, where now $\mathbf{U}=\mathbf{U}(x, t)$. We then apply the same far-field expansion of this equation as in [2], by defining

$$
x=a h^{-5}+h^{-1} y, \quad \tau=h^{\sigma} t, \quad \mathbf{U}_{n}(t)=\mathbf{U}_{0}(y, \tau)+h^{2} \mathbf{U}_{1}(y, \tau)+h^{4} \mathbf{U}_{2}(y, \tau)+\ldots
$$

The initial expansion of this leads to

$$
\begin{aligned}
2 h^{-5}\left(a+h^{4} y\right)= & 2 h^{\sigma+1}\left(\mathbf{U}_{0, \tau}+h^{2} \mathbf{U}_{1, \tau}+h^{4} \mathbf{U}_{2, \tau}+\ldots\right)\left(\mathbf{U}_{0, y}+h^{2} \mathbf{U}_{1, y}+h^{4} \mathbf{U}_{2, y}+\frac{1}{6} h^{2} \mathbf{U}_{0, y y y}\right. \\
& \left.+\frac{1}{6} h^{4} \mathbf{U}_{1, y y y}+\frac{1}{120} h^{4} \mathbf{U}_{0, y y y y y}+\ldots\right),
\end{aligned}
$$

thus we choose $\sigma=-6$.

We now introduce a long timescale $(T)$ at the same time assuming that the leading order dynamics are governed by a travelling wave; thus we define

$$
z=y-a c \tau, \quad T=h^{2} \tau, \quad \partial_{\tau}=\partial_{\tau}-a c \partial_{z}+h^{2} \partial_{T}, \quad y=z+h^{-2} a c T,
$$

where $h \ll 1$ is a small parameter, $c$ is a wavespeed that will be determined later.

The expansion (3.42) thus becomes

$$
\begin{aligned}
\left(a+h^{4} z+a c T h^{2}\right) \mathbf{I}= & \left(\mathbf{U}_{0, \tau}-a c \mathbf{U}_{0, z}+h^{2} \mathbf{U}_{0, T}+h^{2} \mathbf{U}_{1, \tau}-h^{2} a c \mathbf{U}_{1, z}+h^{4} \mathbf{U}_{1, T}+h^{4} \mathbf{U}_{2, \tau}\right. \\
& \left.-h^{4} a c \mathbf{U}_{2, z}+\ldots\right)\left(\mathbf{U}_{0, z}+h^{2} \mathbf{U}_{1, z}+h^{4} \mathbf{U}_{2, z}+\frac{1}{6} h^{2} \mathbf{U}_{0, z z z}\right. \\
& \left.+\frac{1}{6} h^{4} \mathbf{U}_{1, z z z}+\frac{1}{120} h^{4} \mathbf{U}_{0, z z z z z}+\ldots\right) .
\end{aligned}
$$

We assume that $\mathbf{U}_{1}, \mathbf{U}_{2}$ depend only on $(z, T)$ and not on $\tau$.

Expanding (3.44) leads to a sequence of equations at successive powers of $h$, namely

$$
\begin{aligned}
a \mathbf{I}= & \left(\mathbf{U}_{0, \tau}-a c \mathbf{U}_{0, z}\right) \mathbf{U}_{0, z}, \\
a c T \mathbf{I}= & \left(\mathbf{U}_{0, \tau}-a c \mathbf{U}_{0, z}\right)\left(\mathbf{U}_{1, z}+\frac{1}{6} \mathbf{U}_{0, z z z}\right)+\left(\mathbf{U}_{0, T}-a c \mathbf{U}_{1, z}\right) \mathbf{U}_{0, z}, \\
z \mathbf{I}= & \left(\mathbf{U}_{0, \tau}-a c \mathbf{U}_{0, z}\right)\left(\mathbf{U}_{2, z}+\frac{1}{6} \mathbf{U}_{1, z z z}+\frac{1}{120} \mathbf{U}_{0, z z z z}\right) \\
& +\left(\mathbf{U}_{0, T}-a c \mathbf{U}_{1, z}\right)\left(\mathbf{U}_{1, z}+\frac{1}{6} \mathbf{U}_{0, z z z}\right)+\left(\mathbf{U}_{1, T}-a c \mathbf{U}_{2, z}\right) \mathbf{U}_{0, z} .
\end{aligned}
$$

Solving the leading order terms gives

$$
\mathbf{U}_{0}=z \mathbf{K}+\tau \mathbf{M}+\mathbf{L}(T), \quad \mathbf{M}=a c \mathbf{K}+a \mathbf{K}^{-1},
$$

where $\mathbf{K}$ is an arbitrary constant matrix and $\mathbf{L}$ is an arbitrary function of $T$. To allow the generality of solutions obtained at higher order, we take $\mathbf{K}=k \mathbf{I}$, then $\mathbf{M}=a\left(1+c k^{2}\right) \mathbf{I} / k$.

Using (3.48) to simplify (3.46), we find

$$
a c T k^{-1} \mathbf{I}=a k^{-2} \mathbf{U}_{1, z}+\mathbf{L}^{\prime}(T)-a c \mathbf{U}_{1, z} .
$$

Our aim here is to find the speed, $c$, leaving $\mathbf{U}_{1}$ to be determined by higher order equations. Thus we define

$$
c=1 / k^{2}, \quad \mathbf{L}^{\prime}(T)=a c k^{-1} T \mathbf{I}, \quad \text { so that } \quad \mathbf{L}(T)=\frac{1}{2} a c k^{-1} T^{2} \mathbf{I}+\mathbf{C} .
$$

Considering now the next order terms, namely (3.47), and, using (3.48) to simplify, we find

$$
z k \mathbf{I}=a \mathbf{U}_{2, z}+\frac{1}{6} a \mathbf{U}_{1, z z z}+k \mathbf{L}^{\prime}(T) \mathbf{U}_{1, z}-a c k \mathbf{U}_{1, z} \mathbf{U}_{1, z}+k^{2} \mathbf{U}_{1, T}-a c k^{2} \mathbf{U}_{2, z}
$$

which can be simplified by using $c=k^{-2}$ to give a perturbed matrix potential $\mathrm{KdV}$ equation

$$
k^{2} \mathbf{U}_{1, T}-a c k \mathbf{U}_{1, z}^{2}+\frac{1}{6} a \mathbf{U}_{1, z z z}=k z \mathbf{I}-a c T \mathbf{U}_{1, z} .
$$

This equation has the form of a forced matrix potential KdV system. The forcing terms can be removed by the transformation

$$
\mathbf{U}_{1}(z, T)=\frac{z T}{k} \mathbf{I}+\frac{1}{c k} \mathbf{V}(\xi, \theta), \quad \xi=z+\frac{a c T^{2}}{2 k^{2}}, \quad \theta=\frac{a T}{6 k^{2}},
$$

to leave the matrix potential $\mathrm{KdV}$ system

$$
\mathbf{V}_{\theta}-6 \mathbf{V}_{\xi}^{2}+\mathbf{V}_{\xi \xi \xi}=\mathbf{0}
$$




\subsection{Asymptotic reduction of the nonautonomous $\mathrm{W}$-equation to matrix $\mathrm{KdV}$}

We outline asymptotic reduction of the nonautonomous differential-difference equation for $\mathbf{W}_{n}$ to the matrix $\mathrm{KdV}$ equation. In

$$
\mathbf{W}_{n, t}=-\mathbf{W}_{n}\left[(2 n+1) \mathbf{W}_{n+1}-(2 n-3) \mathbf{W}_{n-1}\right] \mathbf{W}_{n},
$$

we introduce a shifted independent variable, $x=n-\frac{1}{2}$, to symmetrise the discrete difference, and then expand

$$
\mathbf{W}_{t}(x, t)=-2 \mathbf{W}(x, t)[(x+1) \mathbf{W}(x+1, t)-(x-1) \mathbf{W}(x-1, t)] \mathbf{W}(x, t),
$$

in the far field $(x \gg 1)$, using the new variables given by

$$
x=a h^{-5}+y h^{-1}, \quad t=h^{4} \tau, \quad h \ll 1, \quad \mathbf{W}(x, t)=\widetilde{\mathbf{W}}(y, \tau) .
$$

Taking the continuum limit of (3.56), we find the first few terms, up to $O\left(h^{4}\right)$ as

$$
\widetilde{\mathbf{W}}_{\tau}=-4 \widetilde{\mathbf{W}}\left[a \widetilde{\mathbf{W}}_{y}+\frac{1}{6} a h^{2} \widetilde{\mathbf{W}}_{y y y}+\frac{1}{120} h^{4} \widetilde{\mathbf{W}}_{\text {yyyyyy }}+h^{4} y \widetilde{\mathbf{W}}_{y}+h^{4} \widetilde{\mathbf{W}}\right] \widetilde{\mathbf{W}}
$$

We transform to a moving coordinate frame given by

$$
z=y-c \tau, \quad T=h^{2} \tau,
$$

where $T$ is a long timescale. We assume that there is no $\tau$-dependence in $\widetilde{\mathbf{W}}_{0}, \widetilde{\mathbf{W}}_{1}$ or $\widetilde{\mathbf{W}}_{2}$ used below, so $(y, \tau)$ are replaced by $(z, T)$ via $y=z+c T / h^{2}$, and $\partial_{\tau}=-c \partial_{z}+h^{2} \partial_{T}$. We assume $\mathbf{W}(x, t)=\widetilde{\mathbf{W}}(y, \tau)$ can be described by an asymptotic expansion of the form

$$
\mathbf{W}(x, t)=\widetilde{\mathbf{W}}(y, \tau)=\widetilde{\mathbf{W}}_{0}(z, T)+h^{2} \widetilde{\mathbf{W}}_{1}(z, T)+h^{4} \widetilde{\mathbf{W}}_{2}(z, T)+\ldots
$$

Hence (3.58) becomes, up to order $O\left(h^{4}\right)$

$$
\begin{aligned}
& h^{2} \widetilde{\mathbf{W}}_{0, T}-c \widetilde{\mathbf{W}}_{0, z}+h^{4} \widetilde{\mathbf{W}}_{1, T}-c h^{2} \widetilde{\mathbf{W}}_{1, z}-c h^{4} \widetilde{\mathbf{W}}_{2, z} \\
= & -4\left[\widetilde{\mathbf{W}}_{0}+h^{2} \widetilde{\mathbf{W}}_{1}+h^{4} \widetilde{\mathbf{W}}_{2}\right]\left[a \widetilde{\mathbf{W}}_{0, z}+a h^{2} \widetilde{\mathbf{W}}_{1, z}+a h^{4} \widetilde{\mathbf{W}}_{2, z}+\frac{1}{6} a h^{2} \widetilde{\mathbf{W}}_{0, z z z}+\frac{1}{6} a h^{4} \widetilde{\mathbf{W}}_{1, z z z}\right. \\
& \left.+\frac{1}{120} a h^{4} \widetilde{\mathbf{W}}_{0, z z z z z}+h^{4}\left(z+c T h^{-2}\right) \widetilde{\mathbf{W}}_{0, z}+c T h^{4} \widetilde{\mathbf{W}}_{1, z}+h^{4} \widetilde{\mathbf{W}}_{0}\right]\left[\widetilde{\mathbf{W}}_{0}+h^{2} \widetilde{\mathbf{W}}_{1}+h^{4} \widetilde{\mathbf{W}}_{2}\right] .
\end{aligned}
$$

We now consider terms at each order of $h$, finding at leading order

$$
-c \widetilde{\mathbf{W}}_{0, z}=-4 a \widetilde{\mathbf{W}}_{0} \widetilde{\mathbf{W}}_{0, z} \widetilde{\mathbf{W}}_{0} .
$$

We take the solution $\widetilde{\mathbf{W}}_{0, z}=\mathbf{0}$, which implies $\widetilde{\mathbf{W}}_{0}=\mathbf{L}(T)$.

At next order, we find

$$
\widetilde{\mathbf{W}}_{0, T}-c \widetilde{\mathbf{W}}_{1, z}=-4 a \widetilde{\mathbf{W}}_{0} \widetilde{\mathbf{W}}_{0, z} \widetilde{\mathbf{W}}_{1}-4 a \widetilde{\mathbf{W}}_{1} \widetilde{\mathbf{W}}_{0, z} \widetilde{\mathbf{W}}_{0}-4 \widetilde{\mathbf{W}}_{0}\left[a \widetilde{\mathbf{W}}_{1, z}+\frac{1}{6} a \widetilde{\mathbf{W}}_{0, z z z}+c T \widetilde{\mathbf{W}}_{0, z}\right] \widetilde{\mathbf{W}}_{0}
$$

which simplifies to

$$
\mathbf{L}^{\prime}(T)-c \widetilde{\mathbf{W}}_{1, z}=-4 a \widetilde{\mathbf{W}}_{0} \widetilde{\mathbf{W}}_{1, z} \widetilde{\mathbf{W}}_{0} .
$$

From this equation we choose $\mathbf{L}^{\prime}(T)=\mathbf{0}$, and then if $\widetilde{\mathbf{W}}_{0}=\mathbf{L}=k \mathbf{I}$ for some arbitrary constant, $k$, $\widetilde{\mathbf{W}}_{1}(z, T)$ is arbitrary, with the speed $c$ being given by $c=4 a k^{2}$.

At the following order, from (3.61) we have

$$
\begin{aligned}
\widetilde{\mathbf{W}}_{1, T}-c \widetilde{\mathbf{W}}_{2, z}= & -4 a \widetilde{\mathbf{W}}_{2} \widetilde{\mathbf{W}}_{0, z} \widetilde{\mathbf{W}}_{0}-4 a \widetilde{\mathbf{W}}_{0} \widetilde{\mathbf{W}}_{0, z} \widetilde{\mathbf{W}}_{2}-4 a \widetilde{\mathbf{W}}_{1} \widetilde{\mathbf{W}}_{0, z} \widetilde{\mathbf{W}}_{1} \\
& -4 \widetilde{\mathbf{W}}_{0}\left[a \widetilde{\mathbf{W}}_{1, z}+\frac{1}{6} a \widetilde{\mathbf{W}}_{0, z z z}+c T \widetilde{\mathbf{W}}_{0, z}\right] \widetilde{\mathbf{W}}_{1}-4 \widetilde{\mathbf{W}}_{1}\left[a \widetilde{\mathbf{W}}_{1, z}+\frac{1}{6} a \widetilde{\mathbf{W}}_{0, z z z}+c T \widetilde{\mathbf{W}}_{0, z}\right] \widetilde{\mathbf{W}}_{0} \\
& -4 \widetilde{\mathbf{W}}_{0}\left[a \widetilde{\mathbf{W}}_{2, z}+\frac{1}{6} a \widetilde{\mathbf{W}}_{1, z z z}+c T \widetilde{\mathbf{W}}_{1, z}+\frac{1}{120} a \widetilde{\mathbf{W}}_{0, z z z z z}+z \widetilde{\mathbf{W}}_{0, z}+\widetilde{\mathbf{W}}_{0}\right] \widetilde{\mathbf{W}}_{0},
\end{aligned}
$$

which can be simplified to

$$
\widetilde{\mathbf{W}}_{1, T}=-4 a k \widetilde{\mathbf{W}}_{1, z} \widetilde{\mathbf{W}}_{1}-4 a k \widetilde{\mathbf{W}}_{1} \widetilde{\mathbf{W}}_{1, z}-\frac{2}{3} a k^{2} \widetilde{\mathbf{W}}_{1, z z z}-16 a k^{4} T \widetilde{\mathbf{W}}_{1, z}-4 k^{3} \mathbf{I},
$$

using the leading order solution $\widetilde{\mathbf{W}}_{0}=k \mathbf{I}$, with $c=4 a k^{2}$. This has the form of a forced matrix $\mathrm{KdV}$ equation which can be mapped onto the standard matrix $\mathrm{KdV}$ equation

$$
\mathbf{V}_{\theta}=3\left(\mathbf{V} \mathbf{V}_{\xi}+\mathbf{V}_{\xi} \mathbf{V}\right)+\mathbf{V}_{\xi \xi \xi}
$$

by the transformation

$$
\widetilde{\mathbf{W}}_{1}(z, T)=-4 k^{3} T \mathbf{I}+\frac{1}{2} k \mathbf{V}(\xi, \theta), \quad \xi=z+8 a k^{4} T^{2}, \quad \theta=-\frac{2}{3} a k^{2} T .
$$




\section{Conclusions}

We have considered the matrix lattice equation $\mathbf{U}_{n, t}\left(\mathbf{U}_{n+1}-\mathbf{U}_{n-1}\right)=g(n) \mathbf{I}$, in both its autonomous $(g(n)=2)$ and nonautonomous $(g(n)=2 n-1)$ forms. For each of these cases we have proved integrability by constructing a Miura map to a corresponding integrable equation in $\mathbf{W}_{n}$, that is, equations (2.3) and (3.3) respectively. We have also sought transformations from these equations in $\mathbf{W}_{n}$ to corresponding autonomous and nonautonomous matrix Volterra systems, in two matrix dependent variables $\mathbf{Y}_{n}$ and $\mathbf{Z}_{n}$. However, in general, the entries of $\mathbf{Y}_{n}$ and $\mathbf{Z}_{n}$ will be subject to consistency conditions. Such constraints would seem difficult to embody in any Lax pairs wherein the only dependent variables are matrices.

This then led us to consider special classes of matrices $\mathbf{Y}_{n}$ and $\mathbf{Z}_{n}$ defined in terms of properties of the matrices $\mathbf{W}_{n}$. The case of symmetric matrices $\mathbf{W}_{n}$ still requires constraints on the entries of $\mathbf{Y}_{n}$ and $\mathbf{Z}_{n}$ to be taken into account. However, in the case where all matrices $\mathbf{W}_{n}, \mathbf{W}_{m}$ commute — which implies that $\mathbf{Z}_{n}=\mathbf{Y}_{n}$ and that all $\mathbf{Y}_{n}, \mathbf{Y}_{m}$ also commute - we obtain integrable coupled systems of autonomous and nonautonomous lattice equations, along with Miura maps and Lax pairs. Such coupled systems, Miura maps and Lax pairs can be obtained beginning with any size of square matrix; here we have considered in detail the example of $2 \times 2$ matrices, with the structure of commuting $3 \times 3$ matrices being explored in Appendix A. These integrable coupled systems are multicomponent analogs of the scalar equation (1.5) and the Volterra equation (1.7), in the autonomous case, and of the scalar equation (3.8) and the nonisospectral Volterra equation (3.10), in the nonautonomous case. In the autonomous case, the Lax pairs for the multicomponent systems are obtained from Lax pairs for matrix equations in $\mathbf{W}_{n}$ and $\mathbf{Y}_{n}$ under a commutativity assumption, and it is the link we obtain between these equations in commuting matrices that yields multicomponent Miura transformations. Similarly in the nonautonomous case. It is worth noting that in the nonautonomous case, even the relation between the scalar equation (3.8) and the nonisospectral Volterra equation (3.10) via the transformation (3.9) - that is, corresponding to one component - seems to be new. Moreover, the nonisospectral matrix Volterra system (3.20), (3.21) and its Lax pair - which under a commutativity assumption gives rise to Lax pairs for multicomponent nonautonomous lattice equations of Volterra type - also appear to be new.

In addition, we have considered asymptotic reductions to matrix PDEs. In both the autonomous and nonautonomous cases, the $\mathbf{U}$-equation admits such a reduction to the matrix potential $\mathrm{KdV}$ equation, and the $\mathbf{W}$-equation to the matrix $\mathrm{KdV}$ equation. Finally, we have shown how the autonomous matrix lattice systems can be obtained as limiting cases of the nonautonomous matrix lattice systems. In future work, we will explore further the systems discussed here, including for example their connections with autonomous and nonautonomous matrix lattices of Toda type.

\section{Acknowledgment}

The authors are grateful to the Ministry of Economy and Competitiveness of Spain for supporting their work through contract MTM2016-80276-P (AEI/FEDER, EU).

\section{Appendix A. General classes of commuting matrices}

\section{Appendix A.1. Commuting $2 \times 2$ matrices}

To construct the most general class of $2 \times 2$ matrices which commute under multiplication, let us consider

$$
\left(\begin{array}{ll}
a & b \\
c & d
\end{array}\right)\left(\begin{array}{ll}
\widehat{a} & \widehat{b} \\
\widehat{c} & \widehat{d}
\end{array}\right)=\left(\begin{array}{ll}
\widehat{a} & \widehat{b} \\
\widehat{c} & \widehat{d}
\end{array}\right)\left(\begin{array}{ll}
a & b \\
c & d
\end{array}\right) .
$$

The elements on the leading diagonal imply $b \widehat{c}=\widehat{b} c$, or, equivalently, $\widehat{c} \widehat{b}=c / b$, thus if $c=K b$, we necessarily have $\widehat{c}=K \vec{b}$, with the same constant $K$. Similarly, the upper right element of the products implies $\widehat{a} b+\widehat{b} d=\widehat{a} \widehat{b}+b \widehat{d}$, so we may set

$$
\frac{\widehat{d}-\widehat{a}}{\widehat{b}}=\frac{d-a}{b}=M,
$$


for some constant $M$. It can be confirmed that the equation from the lower left element is automatically satisfied. Thus the most general class of commuting $2 \times 2$ matrices is

$$
\left(\begin{array}{cc}
a & b \\
K b & a+M b
\end{array}\right)
$$

where $a, b$ can be chosen freely and $K, M$ are fixed.

We note that this class of matrices is closed under multiplication, that is, the product of two matrices of the form given in (A3) has this same form (see (2.32) and (2.33)):

$$
\left(\begin{array}{cc}
a & b \\
K b & a+M b
\end{array}\right)\left(\begin{array}{cc}
\widehat{a} & \widehat{b} \\
K \widehat{b} & \widehat{a}+M \widehat{b}
\end{array}\right)=\left(\begin{array}{cc}
(a \widehat{a}+K \widehat{b} \vec{b}) & \widehat{a b}+b \widehat{a}+M b \widehat{b} \\
K(\widehat{a} b+a \widehat{b}+M b \widehat{b}) & (a \widehat{a}+K \vec{b})+M(\widehat{a} b+a \widehat{b}+M b \widehat{b})
\end{array}\right)
$$

This class of matrices is also closed under linear combination. These facts are important because they assure the consistency of the coupled systems of autonomous and nonautonomous lattice equations derived in Sections 2.2.2 and 3.3.2. (In fact, they assure consistency of the coupled lattice equations derived from a matrix lattice equation, where the evolution of the matrix dependent variable is polynomial in shifts of the same, when the matrices are assumed to be of this form.)

\section{Appendix A.2. Commuting $3 \times 3$ matrices} by

Following the same procedure, we obtain the general class of commuting $3 \times 3$ matrices given

$$
\left(\begin{array}{ccc}
a & b & c \\
k_{7} b+k_{8} c & a+k_{4} b+k_{5} c & k_{1} b+k_{2} c \\
k_{8} b+k_{9} c & k_{5} b+k_{6} c & a+k_{2} b+k_{3} c
\end{array}\right)
$$

where $a, b, c$ can be chosen freely, $k_{j}(1 \leq j \leq 6)$ are fixed, and $k_{j}(7 \leq j \leq 9)$ are given by

$$
k_{7}=k_{1}\left(k_{5}-k_{3}\right)+k_{2}\left(k_{2}-k_{4}\right), \quad k_{8}=k_{6} k_{1}-k_{5} k_{2}, \quad k_{9}=k_{5}\left(k_{5}-k_{3}\right)+k_{6}\left(k_{2}-k_{4}\right) .
$$

Once again, this class of matrices is closed under multiplication, that is, the product of two matrices of the form given in (A5) - one given in terms of $a, b, c$ and the second in terms of $\widehat{a}, \widehat{b}, \widehat{c}$ — has this same form, given by

$$
\left(\begin{array}{ccc}
r & s & t \\
k_{7} s+k_{8} t & r+k_{4} s+k_{5} t & k_{1} s+k_{2} t \\
k_{8} s+k_{9} t & k_{5} s+k_{6} t & r+k_{2} s+k_{3} t
\end{array}\right)
$$

where

$$
\begin{aligned}
& \left.r=k_{7} b \widehat{b}+k_{8} \widehat{(b c}+b \widehat{c}\right)+k_{9} \widehat{c c}+a \widehat{a}, \\
& s=k_{4} \overrightarrow{b b}+k_{5}(\widehat{b} c+b \widehat{c})+k_{6} \widehat{c c}+\widehat{a} b+a \widehat{b}, \\
& t=k_{1} b \widehat{b b}+k_{2}(\widehat{b} c+b \widehat{c})+k_{3} \widehat{c c}+\widehat{a} c+a \widehat{c} .
\end{aligned}
$$

This class of matrices is also closed under linear combination. These facts are important because they assure the consistency of any coupled systems of autonomous and nonautonomous lattice equations derived as explained in Sections 2.2.2 and 3.3.2 using $3 \times 3$ commuting matrices of the form given in (A5).

A second class of commuting $3 \times 3$ matrices is given by

$$
\left(\begin{array}{ccc}
a & b & k_{0} b \\
k_{1} b & a+k_{2} b & k_{3} b \\
k_{4} b & k_{5} b & a+k_{6} b
\end{array}\right)
$$


where $a, b$ can be chosen freely and $k_{j}(0 \leq j \leq 6)$ are fixed. However, in the general case, matrices of this form are not closed under multiplication. They are closed under multiplication in the special case where

$k_{1}=k_{0} k_{5}\left(k_{0} k_{5}-k_{6}\right)-k_{6}\left(k_{2}+k_{0} k_{5}-k_{6}\right)+k_{0} k_{2} k_{5}, \quad k_{3}=k_{0}\left(k_{2}+k_{0} k_{5}-k_{6}\right), \quad k_{4}=k_{5}\left(k_{0} k_{5}-k_{6}\right)$.

They are also closed under linear combination. Thus in this special case (A10), the consistency of any coupled systems of autonomous and nonautonomous lattice equations derived as explained in Sections 2.2.2 and 3.3.2 is assured.

\section{Appendix B. Examples of coupled systems in the nonautonomous case}

Here we return to the nonautonomous systems (3.26) and (3.27), and quote the results corresponding to the standard examples - elliptic/complex, dual/shear, hyperbolic/cyclic, and generalised hyperbolic - of commuting $2 \times 2$ matrices, as referred to at the end of Section 3.3.2. These systems are

$$
\begin{aligned}
a_{n, t}= & -\left(a_{n}^{2}+K b_{n}^{2}\right)\left[(2 n+1) a_{n+1}-(2 n-3) a_{n-1}\right]-K b_{n}\left(2 a_{n}+M b_{n}\right)\left[(2 n+1) b_{n+1}-(2 n-3) b_{n-1}\right], \\
b_{n, t}= & -\left(a_{n}^{2}+K b_{n}^{2}\right)\left[(2 n+1) b_{n+1}-(2 n-3) b_{n-1}\right]-b_{n}\left(2 a_{n}+M b_{n}\right)\left[(2 n+1) a_{n+1}-(2 n-3) a_{n-1}\right] \\
& -M b_{n}\left(2 a_{n}+M b_{n}\right)\left[(2 n+1) b_{n+1}-(2 n-3) b_{n-1}\right],
\end{aligned}
$$

and

$$
\begin{aligned}
r_{n, t}= & r_{n}\left[(2 n-3) r_{n-1}-(2 n+3) r_{n+1}-2 r_{n}\right]+K s_{n}\left[(2 n-3) s_{n-1}-(2 n+3) s_{n+1}-2 s_{n}\right], \\
s_{n, t}= & r_{n}\left[(2 n-3) s_{n-1}-(2 n+3) s_{n+1}-2 s_{n}\right]+s_{n}\left[(2 n-3) r_{n-1}-(2 n+3) r_{n+1}-2 r_{n}\right] \\
& +M s_{n}\left[(2 n-3) s_{n-1}-(2 n+3) s_{n+1}-2 s_{n}\right] .
\end{aligned}
$$

The elliptic/complex case is given by $M=0, K=-1$, which leads to the governing equations for $\left(a_{n}, b_{n}\right),\left(r_{n}, s_{n}\right)$ as

$$
\begin{aligned}
& a_{n, t}=-\left(a_{n}^{2}-b_{n}^{2}\right)\left[(2 n+1) a_{n+1}-(2 n-3) a_{n-1}\right]+2 a_{n} b_{n}\left[(2 n+1) b_{n+1}-(2 n-3) b_{n-1}\right], \\
& b_{n, t}=-\left(a_{n}^{2}-b_{n}^{2}\right)\left[(2 n+1) b_{n+1}-(2 n-3) b_{n-1}\right]-2 a_{n} b_{n}\left[(2 n+1) a_{n+1}-(2 n-3) a_{n-1}\right],
\end{aligned}
$$

and

$$
\begin{aligned}
& r_{n, t}=r_{n}\left[(2 n-3) r_{n-1}-(2 n+3) r_{n+1}-2 r_{n}\right]+s_{n}\left[(2 n-3) s_{n-1}-(2 n+3) s_{n+1}-2 s_{n}\right], \\
& s_{n, t}=r_{n}\left[(2 n-3) s_{n-1}-(2 n+3) s_{n+1}-2 s_{n}\right]+s_{n}\left[(2 n-3) r_{n-1}-(2 n+3) r_{n+1}-2 r_{n}\right] .
\end{aligned}
$$

In the dual/shear case with $K=M=0$, the systems for $\left(a_{n}, b_{n}\right),\left(r_{n}, s_{n}\right)$ are

$$
\begin{aligned}
& a_{n, t}=-a_{n}^{2}\left[(2 n+1) a_{n+1}-(2 n-3) a_{n-1}\right], \\
& b_{n, t}=-a_{n}^{2}\left[(2 n+1) b_{n+1}-(2 n-3) b_{n-1}\right]-2 a_{n} b_{n}\left[(2 n+1) a_{n+1}-(2 n-3) a_{n-1}\right], \\
r_{n, t}= & r_{n}\left[(2 n-3) r_{n-1}-(2 n+3) r_{n+1}-2 r_{n}\right], \\
s_{n, t}= & r_{n}\left[(2 n-3) s_{n-1}-(2 n+3) s_{n+1}-2 s_{n}\right]+s_{n}\left[(2 n-3) r_{n-1}-(2 n+3) r_{n+1}-2 r_{n}\right] .
\end{aligned}
$$

The hyperbolic/cyclic case is given by $K=1, M=0$, which leads to

$$
\begin{aligned}
& a_{n, t}=-\left(a_{n}^{2}+b_{n}^{2}\right)\left[(2 n+1) a_{n+1}-(2 n-3) a_{n-1}\right]-2 a_{n} b_{n}\left[(2 n+1) b_{n+1}-(2 n-3) b_{n-1}\right], \\
& b_{n, t}=-\left(a_{n}^{2}+b_{n}^{2}\right)\left[(2 n+1) b_{n+1}-(2 n-3) b_{n-1}\right]-2 a_{n} b_{n}\left[(2 n+1) a_{n+1}-(2 n-3) a_{n-1}\right],
\end{aligned}
$$

and

$$
\begin{aligned}
& r_{n, t}=r_{n}\left[(2 n-3) r_{n-1}-(2 n+3) r_{n+1}-2 r_{n}\right]+s_{n}\left[(2 n-3) s_{n-1}-(2 n+3) s_{n+1}-2 s_{n}\right] \\
& s_{n, t}=r_{n}\left[(2 n-3) s_{n-1}-(2 n+3) s_{n+1}-2 s_{n}\right]+s_{n}\left[(2 n-3) r_{n-1}-(2 n+3) r_{n+1}-2 r_{n}\right]
\end{aligned}
$$


Our generalised hyperbolic case is given by $K=1, M=-2$, with $a_{n}=e_{n}+f_{n}, b_{n}=f_{n}$, which leads to

$$
\begin{aligned}
& e_{n, t}=-\left(e_{n}^{2}+2 f_{n}^{2}\right)\left[(2 n+1) e_{n+1}-(2 n-3) e_{n-1}\right]-4 e_{n} f_{n}\left[(2 n+1) f_{n+1}-(2 n-3) f_{n-1}\right], \\
& f_{n, t}=-\left(e_{n}^{2}+2 f_{n}^{2}\right)\left[(2 n+1) f_{n+1}-(2 n-3) f_{n-1}\right]-2 e_{n} f_{n}\left[(2 n+1) e_{n+1}-(2 n-3) e_{n-1}\right],
\end{aligned}
$$

and, with $r_{n}=y_{n}+z_{n}$ and $s_{n}=z_{n}$, to

$$
\begin{aligned}
& y_{n, t}=y_{n}\left[(2 n-3) y_{n-1}-(2 n+3) y_{n+1}-2 y_{n}\right]+2 z_{n}\left[(2 n-3) z_{n-1}-(2 n+3) z_{n+1}-2 z_{n}\right] \\
& z_{n, t}=y_{n}\left[(2 n-3) z_{n-1}-(2 n+3) z_{n+1}-2 z_{n}\right]+z_{n}\left[(2 n-3) y_{n-1}-(2 n+3) y_{n+1}-2 y_{n}\right] .
\end{aligned}
$$

\section{References}

[1] PR Gordoa, A Pickering. Auto-Bäcklund transformations for a matrix partial differential equation. Phys Lett A, 382, 1908-1915, (2018).

[2] A Pickering, PR Gordoa, JAD Wattis. The second Painlevé equation, a related nonautonomous semidiscrete equation and a limit to the first Painlevé equation: scalar and matrix cases. Physica D, 391, 72-86, (2019).

[3] V Volterra. Leçons sur la Théorie Mathematique de la Lutte pour la Vie (Gauthier-Villars, Paris, 1931).

[4] M Kac \& P van Moerbeke. On an explicitly soluble system of nonlinear differential equations related to certain Toda lattices. Advances Math., 16, 160-169, (1975).

[5] SV Manakov. Complete integrability and stochastization of discrete dynamical systems. Zh. Eksp. Teor. Fiz., 67, 543-555, (1974); Sov. Phys. JETP, 40, 269-274, (1975).

[6] M Wadati. Transformation theories for nonlinear discrete systems. Suppl. Prog. Theor. Phys., 59, 36-63, (1976).

[7] R Hirota \& J Satsuma. N-soliton solutions of nonlinear network equations describing a Volterra system. J. Phys. Soc. Japan, 40, 891-900, (1976).

[8] D Levi, O Ragnisco \& MA Rodríguez. On non-isospectral flows, Painlevé equations and symmetries of differential and difference equations. Teoret. i Matem. Fizika, 93, 473-480, (1992); Theor. Math. Phys., 93, 1409-1414, (1993).

[9] R Yamilov. Construction scheme for discrete Miura transformations. J. Phys. A, 27, 6839-6851, (1994).

[10] J Villarroel, S Chakravarty \& MJ Ablowitz. On a $2+1$ Volterra system. Nonlinearity, 9, 1113-1128, (1996).

[11] XB Hu \& RK Bullough. Bäcklund transformation and nonlinear superposition formula of an extended LotkaVolterra equation. J. Phys. A, 30, 3635-3641, (1997).

[12] Y Kajinaga \& M Wadati. A new derivation of the Bäcklund transformation for the Volterra lattice. J. Phys. Soc. Japan, 67, 2237-2241, (1998).

[13] V.E. Adler, S.I. Svinolupov, R.I. Yamilov. Multi-component Volterra and Toda type integrable equations. Phys Lett A, 254, 24-36, (1999).

[14] WX Ma \& B Fuchssteiner. Algebraic structure of discrete zero curvature equations and master symmetries of discrete evolution equations. J. Math. Phys., 40, 2400-2418, (1999).

[15] Y Kajinaga \& M Wadati. Bäcklund transformation for solutions of the modified Volterra lattice equation. $J$. Phys. Soc. Japan, 68, 51-54, (1999).

[16] ZN Zhu \& HW Tam. Nonisospectral negative Volterra flows and mixed Volterra flows: Lax pairs, infinitely many conservation laws and integrable time discretization. J. Phys. A, 37, 3175-3187, (2004).

[17] PR Gordoa, A Pickering, ZN Zhu. A nonisospectral extension of the Volterra hierarchy to $2+1$ dimensions. $J$. Math. Phys., 46, 103509, (2005).

[18] R Yamilov. Symmetries as integrability criteria for differential difference equations. J Phys A: Math Gen, 39, R541-R623, (2006).

[19] A Pickering, PR Gordoa, JAD Wattis, Behaviour of the extended Volterra lattice, Commun Nonlinear Sci Numer Simulat, 19, 589-600, (2014).

[20] F Muller-Hoissen, O. Chvartatskyi, and K Toda Generalized Volterra lattices: binary Darboux transformations and self-consistent sources. J Geom $\mathcal{F}$ Phys, 113, 226-238, (2017).

[21] JAD Wattis, PR Gordoa, A Pickering. Behaviour of the extended modified Volterra lattice - reductions to generalised mKdV and NLS equations. Commun Nonlin Sci Numer Simul, 65, 98-110, (2018). 